\title{
Article
}

\section{Cognitively Diagnostic Analysis Using the G-DINA Model in R}

\author{
Qingzhou Shi ${ }^{1, *}$, Wenchao Ma ${ }^{1} \mathbb{C}$, Alexander Robitzsch ${ }^{2,3}{ }^{\oplus}$, Miguel A. Sorrel $^{4}\left(\mathbb{C}\right.$ and Kaiwen Man $^{1}$ \\ 1 Department of Educational Studies in Psychology, Research Methodology, and Counseling, University of \\ Alabama, Tuscaloosa, AL 35487, USA; wenchao.ma@ua.edu (W.M.); kman@ua.edu (K.M.) \\ 2 IPN_Leibniz Institute for Science and Mathematics Education, Olshausenstraße 62, 24118 Kiel, Germany; \\ robitzsch@leibniz-ipn.de \\ 3 Centre for International Student Assessment (ZIB), Olshausenstraße 62, 24118 Kiel, Germany \\ 4 Department of Social Psychology and Methodology, Autonomous University of Madrid, 28049 Madrid, Spain; \\ miguel.sorrel@uam.es \\ * Correspondence: qshi7@crimson.ua.edu
}

check for updates

Citation: Shi, Q.; Ma, W.; Robitzsch, A.; Sorrel, M.A.; Man, K. Cognitively Diagnostic Analysis Using the G-DINA Model in R. Psych 2021, 3 , 812-835. https://doi.org/ $10.3390 /$ psych 3040052

Academic Editors: Gongjun $\mathrm{Xu}$ and André Kretzschmar

Received: 16 September 2021

Accepted: 4 December 2021

Published: 8 December 2021

Publisher's Note: MDPI stays neutral with regard to jurisdictional claims in published maps and institutional affiliations.

Copyright: (c) 2021 by the authors. Licensee MDPI, Basel, Switzerland. This article is an open access article distributed under the terms and conditions of the Creative Commons Attribution (CC BY) license (https:// creativecommons.org/licenses/by/ $4.0 /)$.

\begin{abstract}
Cognitive diagnosis models (CDMs) have increasingly been applied in education and other fields. This article provides an overview of a widely used CDM, namely, the G-DINA model, and demonstrates a hands-on example of using multiple R packages for a series of CDM analyses. This overview involves a step-by-step illustration and explanation of performing Q-matrix evaluation, CDM calibration, model fit evaluation, item diagnosticity investigation, classification reliability examination, and the result presentation and visualization. Some limitations of conducting CDM analysis in $\mathrm{R}$ are also discussed.
\end{abstract}

Keywords: cognitive diagnosis models; G-DINA; GDINA R package; CDM R package

\section{Introduction}

Cognitive diagnosis models (CDMs), or diagnostic classification models (DCMs), are psychometric models for classifying individuals into latent classes with unique profiles of attributes. CDMs have increasingly attracted attention in education as they have shown the potential to identify students' strengths and weaknesses and thus aid classroom instruction and learning. In addition to the applications in education [1-4], CDMs have also been applied to other areas recently, such as industrial and organizational psychology [5] and psychiatry [6,7].

Despite the usefulness of CDMs in many fields, software programs for CDM analysis are still lacking. Programs such as Mplus [8,9], JAGS [10,11], and Stan [12,13] have been used for CDM analysis, but they are not without limitations. For example, CDM estimation using these programs often requires advanced coding skills, which may pose a formidable obstacle for CDMs practical application. Also, these general programs typically lack many essential functions, such as those for refining Q-matrix and assessing classification reliability. Recently, several R packages have been developed particularly for CDM analysis. Notably, George et al. [14] introduced the CDM package, and Ma and de la Torre [15] presented the GDINA package and how to apply it for a series of CDM analyses. However, different $R$ packages have different functionality and features, and it remains unclear how these packages can be used in an integrated way for complete CDM analysis. This paper aims to fill this gap by illustrating a comprehensive CDM analysis with a particular emphasis on the use of multiple $\mathrm{R}$ packages under a widely used general CDM-the generalized deterministic input, noisy "and" gate (G-DINA) model [16] with a real dataset. As the first tutorial intended to introduce the state-of-the-art techniques for CDM analyses in the environment of $\mathrm{R}$ via multiple $\mathrm{R}$ packages, this paper will help researchers gain better insight into these packages and conduct CDM analyses in a more principled way. 


\section{The G-DINA Model}

CDMs are latent variable models, where the latent variables may represent skills, abilities, misconceptions, or problem-solving strategies and are referred to as attributes. Attributes are often assumed to have only two statuses, mastery and nonmastery. To conduct a CDM analysis, the item response data and Q-matrix are required. Suppose a test measures $K$ attributes and consists of $J$ items. The $J \times K$ Q-matrix specifies the association between test items and the attributes measured, with $q_{j k}$ being the element on the $j^{\text {th }}$ row and $k^{\text {th }}$ column. If the $k^{\text {th }}$ attribute is assessed by the $j^{\text {th }}$ item, the element $q_{j k}$ equals to 1 ; otherwise, $q_{j k}$ equals to 0 . Let the response of examinee $i$ to item $j$ be denoted by $Y_{i j}$. For each examinee $i$, there is an attribute profile $a_{i}=\left\{a_{i 1}, \ldots, a_{i k}, \ldots, a_{i K}\right\}$ containing $K$ attributes to be inferred. In addition to the item responses and Q-matrix, one must specify the CDM to be used. A CDM consists of the measurement model and the structural model. The former establishes the relationship between the item response and attributes, and the latter specifies the relationship among attributes. To specify a measurement model, one needs to consider the nature of the response data (i.e., binary, ordinal, or nominal), the complexity, and the assumptions of different models. Many measurement models have been discussed in the literature [17]. DINA is a measurement model developed on specific assumptions regarding how attributes affect item responses and thus is often referred to as specific or reduced CDM. In contrast, several other models are referred to as general or saturated models because they have complex parametrizations and subsume many specific models. Examples of general CDMs include the generalized DINA model (G-DINA) [16], the log-linear CDM [18], and the general diagnostic model [19].

Although a simpler model is often preferred if its use could be justified, a saturated model, such as the G-DINA model, may be used to avoid potential model misspecifications. The item response function (IRF) of the G-DINA model [16] is expressed by

$$
g\left[P\left(Y_{i j}=1 \mid a_{l j}^{*}\right)\right]=\delta_{j 0}+\sum_{k=1}^{K_{j}^{*}} \delta_{j k} a_{l k}+\sum_{k^{\prime}=k+1}^{K_{j}^{*}} \sum_{k=1}^{K_{j}^{*}-1} \delta_{j k k^{\prime}} a_{l k} a_{l k k^{\prime}} \ldots+\delta_{j 12 \ldots K_{j}^{*}} \prod_{k=1}^{K_{j}^{*}} a_{l k},
$$

where $g[\cdot]$ represents an identity, logit, or log link function, $\delta_{j 0}$ is the intercept of item $j, \delta_{j k}$ is the main effect of attribute $k, \delta_{j k k^{\prime}}$ is the two-way interaction effect of attributes $k$ and $k^{\prime}$, and $\delta_{j 12 \ldots K_{j}^{*}}$ is the $K_{j}^{*}$-way interaction effect of attributes 1 to $K_{j}^{*}$.

The G-DINA model is an unrestricted, saturated model that can be reduced to many other restricted models by imposing appropriate constraints. In particular, to obtain the deterministic-input, noisy-and-gate (DINA) model [20-22], all terms in Equation (1) except $\delta_{j 0}$ and $\delta_{j 12 \ldots K_{j}^{*}}$ are constrained to be 0 . In this way, the IRF of the DINA model is expressed by

$$
P\left(Y_{i j}=1 \mid a_{l j}^{*}\right)=\delta_{j 0}+\delta_{j 12 \ldots K_{j}^{*}} \prod_{k=1}^{K_{j}^{*}} a_{l k} .
$$

To obtain the deterministic input, noisy-or-gate (DINO) model [6], only the intercept and the main effect of attribute $k$ are kept in the link function of Equation (1), and the IRF of DINO is expressed by

$$
P\left(Y_{i j}=1 \mid a_{l j}^{*}\right)=\delta_{j 0}+\delta_{j k} a_{l k}
$$

where $\delta_{j k}=-\delta_{j k^{\prime} k^{\prime \prime}}=\ldots=(-1)^{K_{j}^{*}+1} \delta_{j 12 \ldots K_{j}^{*}}, k=1, \ldots, K_{j}^{*}, k^{\prime}=1, \ldots, K_{j}^{*}-1$, and $k^{\prime \prime}>k^{\prime}, \ldots, K_{j}^{*}[16]$. In this regard, the number of item parameters for both DINA and DINO models was reduced to just two, regardless of the number of attributes measured. 
To obtain the additive CDM (A-CDM) [16], only the intercept and the main effects in the identity link function of Equation (1) are kept. In this way, the IRF of A-CDM can be expressed by

$$
P\left(Y_{i j}=1 \mid a_{l j}^{*}\right)=\delta_{j 0}+\sum_{k=1}^{K_{j}^{*}} \delta_{j 0} a_{l k} .
$$

The linear logistic model (LLM) [23] is the logit link function of A-CDM, and its IRF can be expressed by

$$
\operatorname{logit}\left[P\left(Y_{i j}=1 \mid a_{l j}^{*}\right)\right]=\delta_{j 0}+\sum_{k=1}^{K_{j}^{*}} \delta_{j 0} a_{l k},
$$

and the reduced reparameterized unified model (R-RUM) [24] is the log-link function of A-CDM, and its IRF is given by

$$
\log \left[P\left(Y_{i j}=1 \mid a_{l j}^{*}\right)\right]=\delta_{j 0}+\sum_{k=1}^{K_{j}^{*}} \delta_{j 0} a_{l k} .
$$

The reduced models presented here can be understood as particular cases of the G-DINA model that accommodate conjunctive or noncompensatory processes (DINA; mastery of all attributes is necessary to have a high probability of success), disjunctive processes (DINO; mastery of one attribute can compensate for the lack of the rest), or additive processes (A-CDM, LLM, and R-RUM; each attribute implies an independent increase in a function of the probability of success).

\section{Overview of the CDM Analyses}

This section will discuss the steps involved in cognitive diagnosis modeling using the G-DINA model, as shown in Figure 1. The development of diagnostic tests and specifications of Q-matrices will not be discussed here; detailed discussions of those can be found in Leighton and Gierl [25], Nichols et al. [26], and Tjoe and de la Torre [19], to name a few.

When the Q-matrix may not be entirely correct, the first step of CDM analysis should be the empirical Q-matrix evaluation, which involves validating the number of attributes and detecting the misidentified elements. To validate the number of attributes, Nájera and colleagues [27] adopted procedures for assessing the dimensionality, which were initially developed for exploratory analysis, often without a provisional Q-matrix. When the number of attributes has been validated, a host of methods have been developed for identifying misspecified elements [28-31]. De la Torre and Minchen [32] recommended employing a saturated CDM when conducting Q-matrix validation to avoid conflating Q-matrix misspecifications with model misspecifications. Also, although statistical procedures could provide some valuable insights into the Q-matrix, the appropriateness of the recommendations of these procedures should be carefully assessed by domain experts. In other words, the Q-matrix validation procedures should be used as a tool to facilitate domain experts developing the Q-matrix.

The second step of CDM analysis often involves model specification. The goal is to determine the measurement model—the model estimating the association between attributes and the observed data-for each item and specify the structural model-the model estimating the association among attributes-for joint attribute distribution. The measurement model can be specified on a priori grounds or determined by statistical procedures. For example, the Wald test and likelihood ratio test have been used to select the measurement model for each item [16,33-35]. Regularized CDMs have also been used to determine each item's most appropriate measurement model [36,37]. It should be noted that monotonicity constraints may need to be imposed because they are often theoretically reasonable and can stabilize the parameter estimation, especially when the sample size is small [38]. 


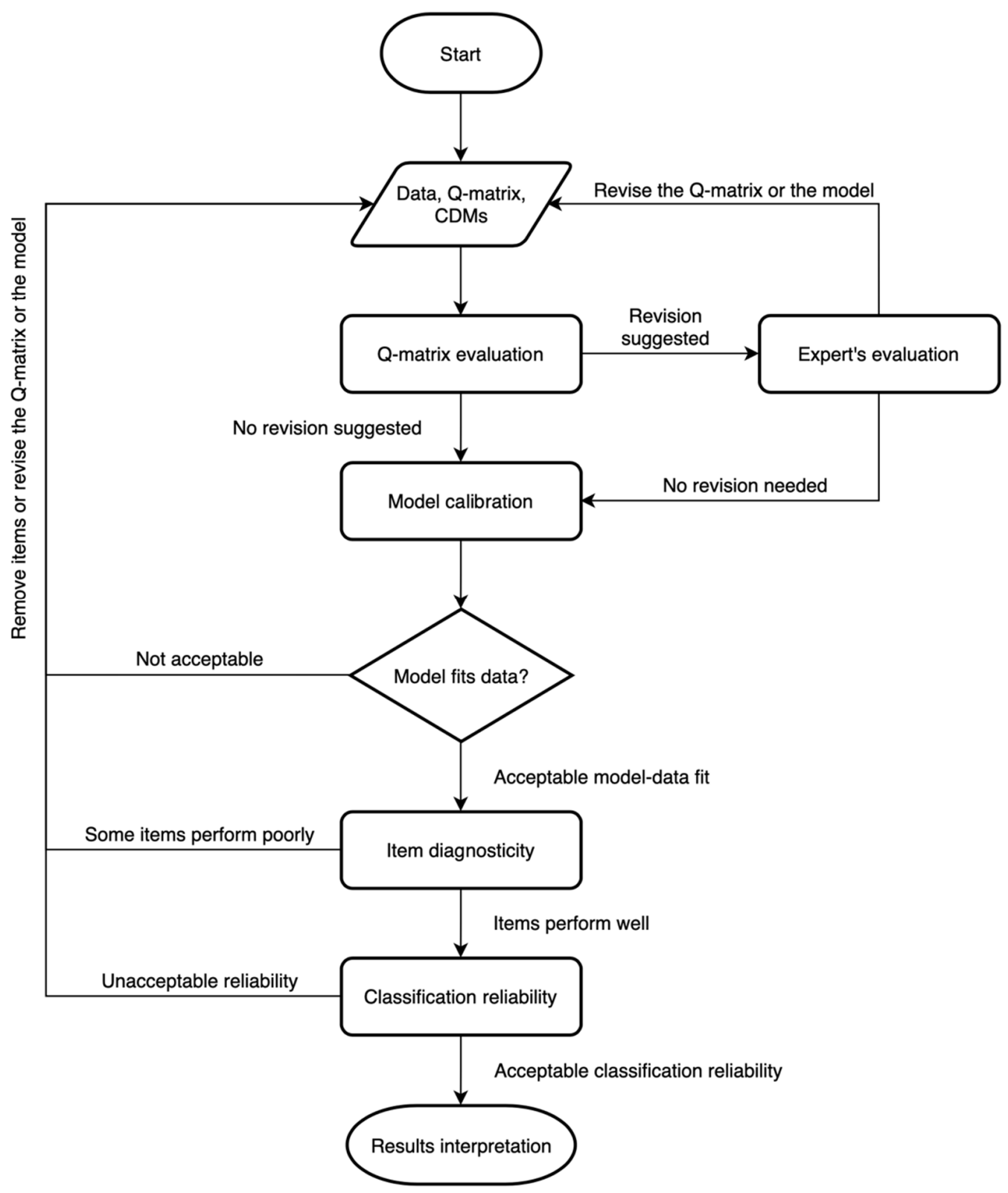

Figure 1. Data analysis diagram using the G-DINA model.

Similarly, the structural model can also be specified based on theories or statistical approaches. For example, when it is believed that attributes have a hierarchical relationship or are related to a common higher-order factor, the structural model should reflect such a belief. The likelihood ratio test can also be performed to compare the saturated structural model with a structural model it subsumes.

The next step of CDM analysis requires assessing model-data fit. Model-data fit can be gauged in an absolute sense, at either the test or item level. The test-level absolute fit evaluation provides information about to what extent the models can fit data well for the whole test, whereas the item-level absolute fit assesses whether or to what extent the model can fit data well for the item. Examples of absolute fit measures include the full information statistics such as Pearson $\chi^{2}$ and limited information statistics such as $\mathrm{M}_{2}$ statistic and RMSEA $_{2}[39,40]$. Models can also be compared using relative fit measures at either test or item level. Examples of measures for relative fit evaluation include information criteria, such as AIC and BIC, and other inferential statistics, such as the Wald test and LR test [41].

When the goodness of fit is adequate, one can interpret model calibration results, including item diagnosticity and person classification reliability. In particular, the item 
characteristic graph showing the probability of success for different latent groups can be displayed in a bar chart. Item discrimination index can also be calculated. Items with poor psychometric properties may need to be removed. In addition to item diagnosticity, test reliability should be investigated. The focus of CDM analysis is often classification, thus, classification accuracy and consistency should be assessed. With satisfactory classification reliability, the final step of CDM analysis is to report person classifications, which could be at the individual level or at an aggregated level for a group of students. It should be noted that CDM analysis may not necessarily be conducted sequentially. For example, the model or the Q-matrix may need to be revised, and some items may need to be removed if the model cannot fit data well.

\section{An Illustration}

This section will use a set of data to illustrate how to use different $R$ packages for CDM analysis.

\subsection{Data and Q-Matrix Preparation}

The dataset we selected the grammar session of the Examination for the Certificate of Proficiency in English (ECPE) to illustrate an example of CDM analysis application, which has been used in several previous studies [9,36,42]. The dataset contains dichotomous responses to 28 items of 2922 students, reflecting their mastery of three grammar rules (attributes): morphosyntactic rules (A1), cohesive rules (A2), and lexical rules (A3). The Q-matrix is given in Table 1.

Table 1. Q-Matrix of the ECPE data.

\begin{tabular}{|c|c|c|c|}
\hline Item & A1 & A2 & A3 \\
\hline 1 & 1 & 1 & 0 \\
\hline 2 & 0 & 1 & 0 \\
\hline 3 & 1 & 0 & 1 \\
\hline 4 & 0 & 0 & 1 \\
\hline 5 & 0 & 0 & 1 \\
\hline 6 & 0 & 0 & 1 \\
\hline 7 & 1 & 0 & 1 \\
\hline 8 & 0 & 1 & 0 \\
\hline 9 & 0 & 0 & 1 \\
\hline 10 & 1 & 0 & 0 \\
\hline 11 & 1 & 0 & 1 \\
\hline 12 & 1 & 0 & 1 \\
\hline 13 & 1 & 0 & 0 \\
\hline 14 & 1 & 0 & 0 \\
\hline 15 & 0 & 0 & 1 \\
\hline 16 & 1 & 0 & 1 \\
\hline 17 & 0 & 1 & 1 \\
\hline 18 & 0 & 0 & 1 \\
\hline 19 & 0 & 0 & 1 \\
\hline 20 & 1 & 0 & 1 \\
\hline 21 & 1 & 0 & 1 \\
\hline 22 & 0 & 0 & 1 \\
\hline 23 & 0 & 1 & 0 \\
\hline 24 & 0 & 1 & 0 \\
\hline 25 & 1 & 0 & 0 \\
\hline 26 & 0 & 0 & 1 \\
\hline 27 & 1 & 0 & 0 \\
\hline 28 & 0 & 0 & 1 \\
\hline
\end{tabular}

Note. A1 = morphosyntactic rules; A2 = cohesive rules, A3 = lexical rules.

As shown in Table 1, the Q-matrix specified the attributes measured by each item. A cell with the value of 1 indicates that the corresponding item measures the corresponding 
attribute, and a cell with the value of 0 indicates the opposite. For example, the attribute vector, or Q-vector, of item 1 is [1], indicating it measures attributes 1 and 2 . One can find the ECPE data and corresponding Q-matrix from CDM [14], GDINA [15], or edmdata [43] R packages.

\subsection{Empirical Q-Matrix Evaluation}

Empirical Q-matrix evaluation involves validating the number of attributes or dimensionality evaluation and detecting misspecified elements in the provisional Q-matrix. Although it usually occurs during the Q-matrix development phase, dimensionality evaluation may provide valuable insight into the structure of the provisional Q-matrix. Dimensionality evaluation can be conducted by the cdmTools [44] package with cdmTools: : paK() and cdmTools: :modelcompK() functions. The cdmTools::paK() function adopts the parallel analysis method by comparing the eigenvalues generated from principal components, Pearson correlations, and mean criterion $[27,45]$ of the randomly resampled correlation matrices and their sample correlation matrices. The argument cor specifies the type of correlations to be used, whose default value is "both", implying using both Pearson and tetrachoric/polychoric correlations. In our code, we define cor = "cor", indicating the Pearson correlations are employed. The number of suggested attributes is extracted by $\$ s u g . K$. As presented in the output below, the suggested number of attributes is 3, which is equal to that of our provisional Q-matrix.

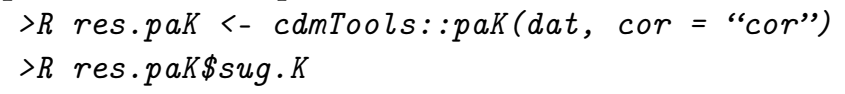

[1] 3

The cdmTools: :modelcompK() function compares several model fit indices of the CDMs fitted with different Q-matrices of a specified number of attributes that are developed through the discrete factor loading method (DFL) [46] and the Hull method [47]. Nájera and colleagues [27] suggested preferring the AIC over other indices. In modelcompK() function, $\operatorname{exploreK}=1: 5$ indicates that Q-matrices with one to five attributes were evaluated.

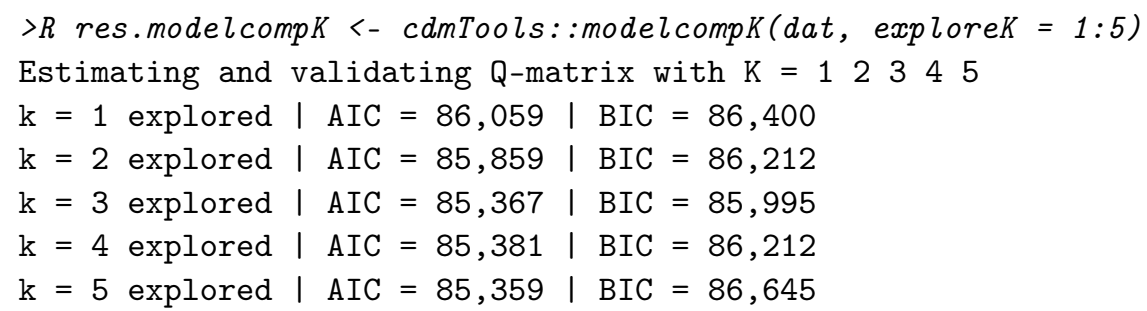

The number of suggested attributes under each model fit index is extracted by $\$ s u g . K$ as well.

$\begin{array}{lllll}>R \text { res.modelcompK\$sug.K } & & \\ \text { AIC } & \text { BIC } & \text { CAIC } & \text { SABIC } & \text { M2 } \\ 5 & 3 & 3 & 3 & 5 \\ \text { M2.p } & \text { SRMSR } & \text { RMSEA2 } & \text { RMSEA2. low } & \text { RMSEA2.high } \\ 5 & 5 & 5 & 5 & 5 \\ \text { sig.item.pairs } & & & \\ 5 & & & \end{array}$

The AIC and BIC values can be plotted across the number of attributes using the $p \operatorname{lot}($ ) function to obtain a direct view of the comparison result. The plots in Figure 2 demonstrate that the tendency change of AIC values and the minimum BIC value are both at $\mathrm{K}=3$. 


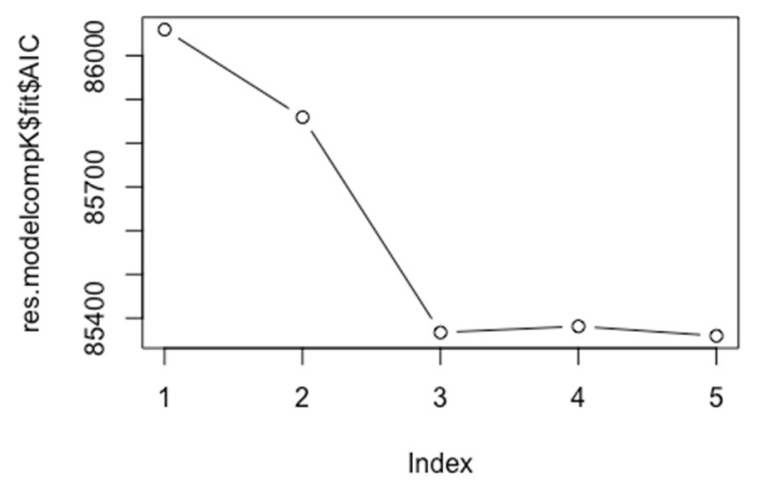

(a)

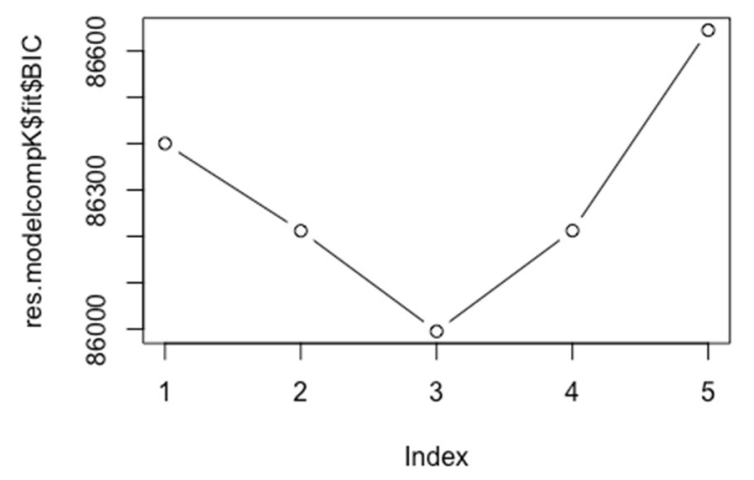

(b)

Figure 2. (a) The scatterplot of AIC; (b) The scatterplot of BIC.

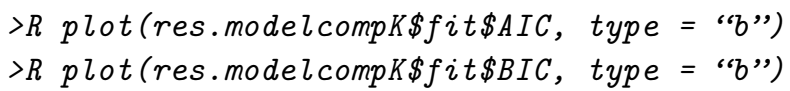

After the number of attributes has been assessed, whether the Q-matrix consists of misspecified elements needs to be examined. Many $\mathrm{R}$ packages provide functions for this purpose. For example, the CDM package implements de la Torre's method [48], and the NPCD package [49] refines the Q-matrix based on Chiu's [50] nonparametric approach. Both methods may be used for mixed DINA and DINO models. The GDINA and cdmTools have functions for Q-matrix validation under the saturated CDMs.

Following the suggestion of de la Torre and Minchen [32], the G-DINA model was employed when conducting Q-matrix validation to avoid conflating Q-matrix misspecifications with model misspecifications. Specifically, the G-DINA model was fitted to the data using the code shown below. The argument mono. constraint is set to TRUE to impose monotonicity constraints to the model, ensuring that the probability of having a correct response of an item will not decrease as the student masters more required attributes. The argument control $=$ list $($ conv. crit $=0.000001)$ indicates that convergence criterion was set to 0.000001 instead of the default value at 0.0001 .

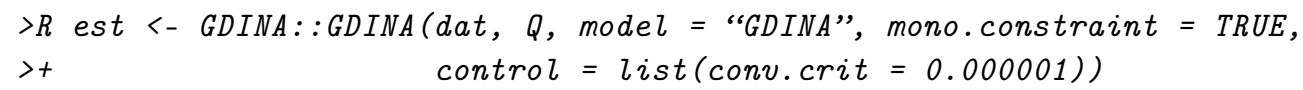

In this paper, the stepwise Wald test [29] was used by specifying method = "wald" in GDINA::Qval() function. Alternatively, the PVAF (i.e., the proportion of variance accounted for) method with fixed or predicted cutoffs can be applied [28] when using this function. In the cdmTools package, Q-matrix validation can be performed with cdmTools: :valQ() function, which implements the Hull method with PVAF or McFadden's pseudo R-squared [47] with various iteration algorithms [31].

$>R$ qv <- GDINA::Qval (GDINA.obj = est, method = "wald")

The suggested Q-matrix based on the stepwise Wald test is presented below. The cells marked with an asterisk are modified according to the validation results. In our case, the q-vector of items 9 and 13 was suggested to be modified.

$\begin{array}{llll}>R & \text { print }(q v) \\ \text { Q-matrix validation } & \text { ba } \\ \text { Suggested } & \text { Q-matrix: } \\ & \text { A1 } & \text { A2 } & \text { A3 } \\ \text { Item 1 } & 1 & 1 & 0 \\ \text { Item 2 } & 0 & 1 & 0 \\ \text { Item 3 } & 1 & 0 & 1 \\ \text { Item 4 } & 0 & 0 & 1 \\ \text { Item 5 } & 0 & 0 & 1 \\ \text { Item 6 } & 0 & 0 & 1\end{array}$




$\begin{array}{llll}\text { Item } 7 & 1 & 0 & 1 \\ \text { Item } 8 & 0 & 1 & 0 \\ \text { Item } 9 & 1 * & 0 & 1 \\ \text { Item } 10 & 1 & 0 & 0 \\ \text { Item } 11 & 1 & 0 & 1 \\ \text { Item } 12 & 1 & 0 & 1 \\ \text { Item } 13 & 1 & 0 & 1 * \\ \text { Item } 14 & 1 & 0 & 0 \\ \text { Item } 15 & 0 & 0 & 1 \\ \text { Item } 16 & 1 & 0 & 1 \\ \text { Item } 17 & 0 & 1 & 1 \\ \text { Item } 18 & 0 & 0 & 1 \\ \text { Item } 19 & 0 & 0 & 1 \\ \text { Item } 20 & 1 & 0 & 1 \\ \text { Item } 21 & 1 & 0 & 1 \\ \text { Item } 22 & 0 & 0 & 1 \\ \text { Item 23 } & 0 & 1 & 0 \\ \text { Item 24 } & 0 & 1 & 0 \\ \text { Item 25 } & 1 & 0 & 0 \\ \text { Item 26 } & 0 & 0 & 1 \\ \text { Item 27 } & 1 & 0 & 0 \\ \text { Item 28 } & 0 & 0 & 1 \\ \text { Note: } & * & \text { denotes a modified element. }\end{array}$

Additionally, the mesa plots were drawn [51] to visualize the PVAF of q-vectors using the code below for Items 9 and 13, as shown in Figure 3. In mesa plots, the $x$-axis represents the q-vectors and the $y$-axis their corresponding PVAF values. The default cutoff value for PVAF (eps) is set to 0.95 out of the range from 0 to 1 . The cutoff can be adjusted according to the researcher's judgment. De la Torre and Ma (2016) recommended the q-vector on the edge of the "mesa" to be considered the correct q-vector for the item. In these mesa plots, the red dots are the original q-vectors. These plots indicate that attributes 3 and 1 each contributed to most of the variance of the item success probabilities of items 9 and 13, respectively, whereas the rest did not contribute much. According to these plots, the original q-vectors [001] and [100] are suggested to be the correct ones instead of the q-vectors [101] in the modified Q-matrix.

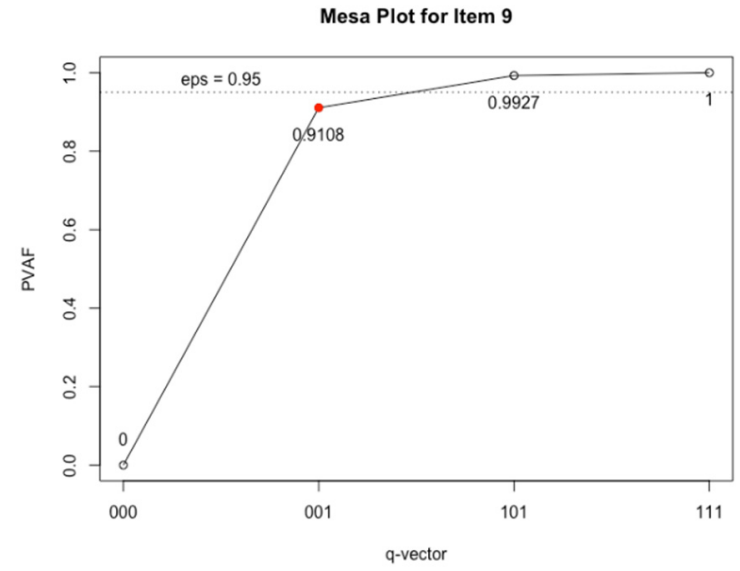

(a)

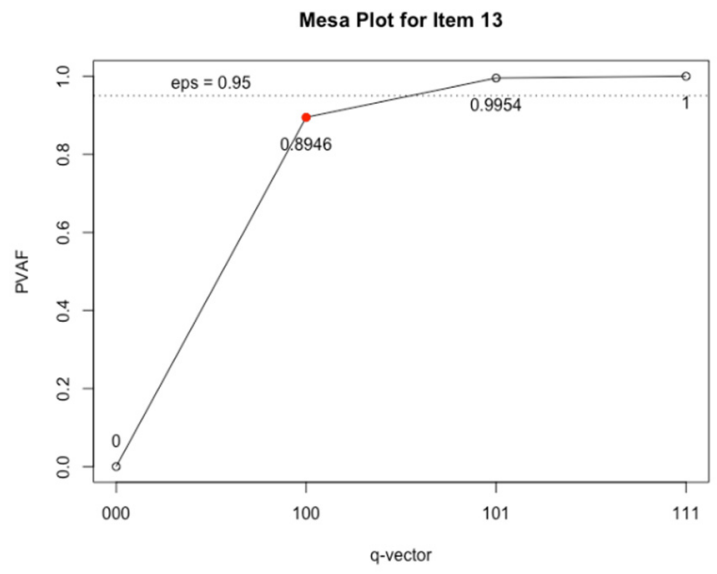

(b)

Figure 3. (a) Mesa plot for item 9; (b) Mesa plot for item 13. 
Please note that, in this section, only some Q-matrix validation methods were discussed when a provisional Q-matrix is available; however, some exploratory methods [52-55] have also been developed to estimate the Q-matrix based on the response data without the need for a provisional Q-matrix. Additionally, different Q-matrix validation methods may produce different recommendations because their performance may be affected by many factors. For example, it has been shown that the stepwise Wald method may prove difficult in converging when the number of attributes is large. As a result, although those recommendations could be valuable for refining the Q-matrix, whether to adopt the suggestions should be contingent on domain experts' judgment and interpretation.

\subsection{CDM Calibration}

After the Q-matrix is finalized, CDMs can fit into the data. Both CDM and GDINA packages can fit the G-DINA model to the data, but they have different default settings. This section will show how the model calibration using one package can be converted to the other package. First, the data is calibrated using the GDINA package with the code below:

$>R$ tol $<-0.000001$

$>R$ GDINA.est <- GDINA::GDINA(dat, Q, model = "GDINA", mono.constraint = TRUE,

$>+\quad$ control $=$ list $($ conv.crit $=$ tol $)$ )

Based on the estimates from the GDINA package, the following code allows fixing the parameters at their estimated values and obtaining an object of "gdina" from the CDM package directly. In particular, item parameter estimates from the GDINA : : GDINA () function of the GDINA package were extracted by GDINA . est $\$$ delta.parm, and the prior probabilities for each latent class for the last E-step of the EM cycle are obtained via GDINA: : extract (GDINA. est, what = "att.prior"). Using the CDM package, the arguments in $C D M:: g d i n a$ () function can be defined with the elements we extracted from GDINA.est. In particular, the arguments delta.fixed and attr.prob.fixed make it possible to fix delta parameters and attribute probabilities, respectively. The argument reduced.skillspace is FALSE, indicating the attribute patterns were not reduced and all possible attribute patterns were included in the estimation [56]. It should be noted that the attribute space needs to be specified using argument skillclasses in that CDM::gdina() and GDINA : :GDINA() functions use different attribute spaces by default. In the code below, the attribute space was defined as att.pattern and then specified in CDM::gdina() function. Because of those settings, GDINA. est and CDM. est contain equivalent estimation results.

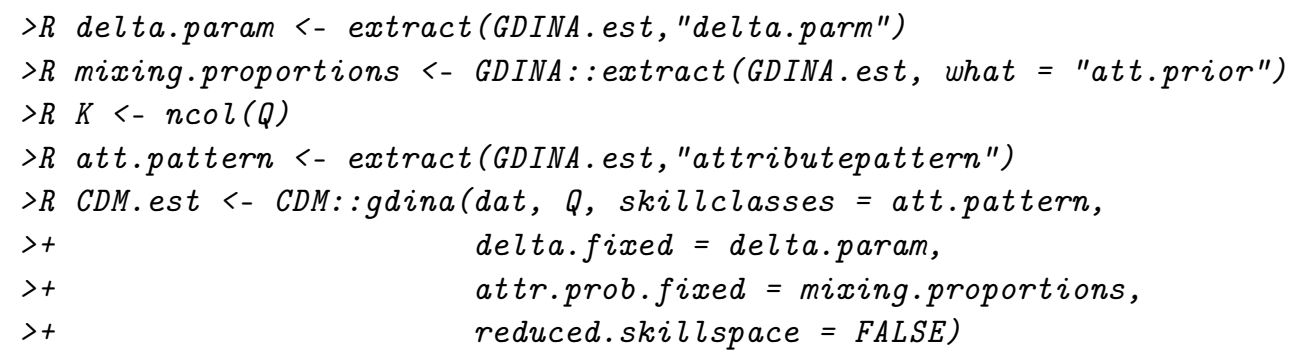

The data can be fit first using the CDM package prior to fixing the parameter estimates in the GDINA package. As shown below, when fitting the data using CDM: :gdina() function, monotonic constraints were imposed using argument mono.constr and set criteria for convergence using arguments conv.crit and dev.crit. It should be noted that when the monotonicity constraints are imposed, a logit link G-DINA model is adopted by default, which is mathematically equivalent to the identity link G-DINA model.

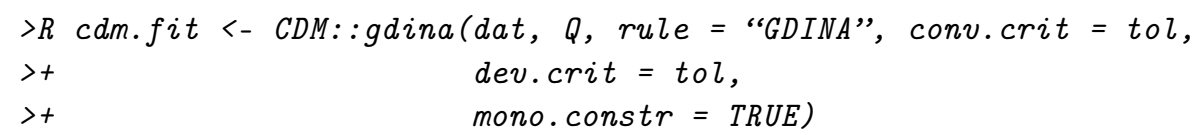


The GDINA : :GDINA () function does not allow fixing delta parameters directly; instead, the item success probabilities can be fixed. The code below extracts the probabilities of success for each reduced attribute profile on each item:

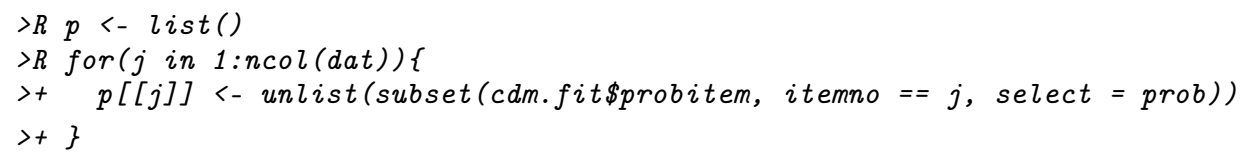

The code below calls GDINA : :GDINA() with several arguments. In particular, the logit link G-DINA model was specified via the arguments model and linkfunc. The attribute space used in the CDM package was extracted via cdm. fit\$attribute.patt.splitted and specified using att.str in GDINA : :GDINA() function. The initial item success probabilities were specified via argument catprob.parm and the initial distribution of latent classes was specified using att.prior. By specifying maxitr $=0$ in argument control, the E-M cycle was disabled, and the initial item success probabilities and distribution of latent classes were used for the final E-step calculation.

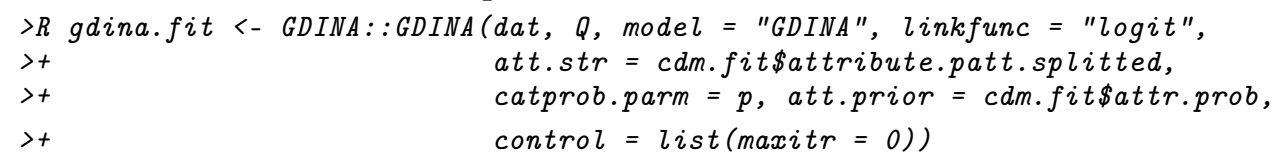

After determining the CDMs and Q-matrix, the assessment is performed to determine whether the parameters of the model can be identified. The function cdmTools: :is. Qid() from the cdmTools package checks model identifiability according to the criteria from Chen and colleagues [53] and Xu and Shang [57]. As shown below, all parameters of the G-DINA model can be identified in this example. Q-matrix in the $Q$ argument, as well as the model that was estimated in the model, need to be provided. Available inputs for the model are "DINA", "DINO", or "others". Here "others" are indicated because of the use of the G-DINA model.

$>R$ cdmTools: :is.Qid( $Q$, model = "others")

So far, the discussion has been focused on how to estimate the G-DINA model using both packages, obtain the equivalent objects between two packages, and assess the identifiability of the G-DINA model globally. In practice, researchers may want to simplify the G-DINA model empirically because it has been shown that reduced models, when used appropriately, can provide better classification results than the G-DINA model [34]. The GDINA package offers a function called GDINA : :modelcomp (), which implements the Wald test and likelihood ratio test for assessing whether the G-DINA model can be reduced to five commonly used reduced models, namely, the DINA model, the DINO model, A-CDM, LLM, and R-RUM, as shown below:

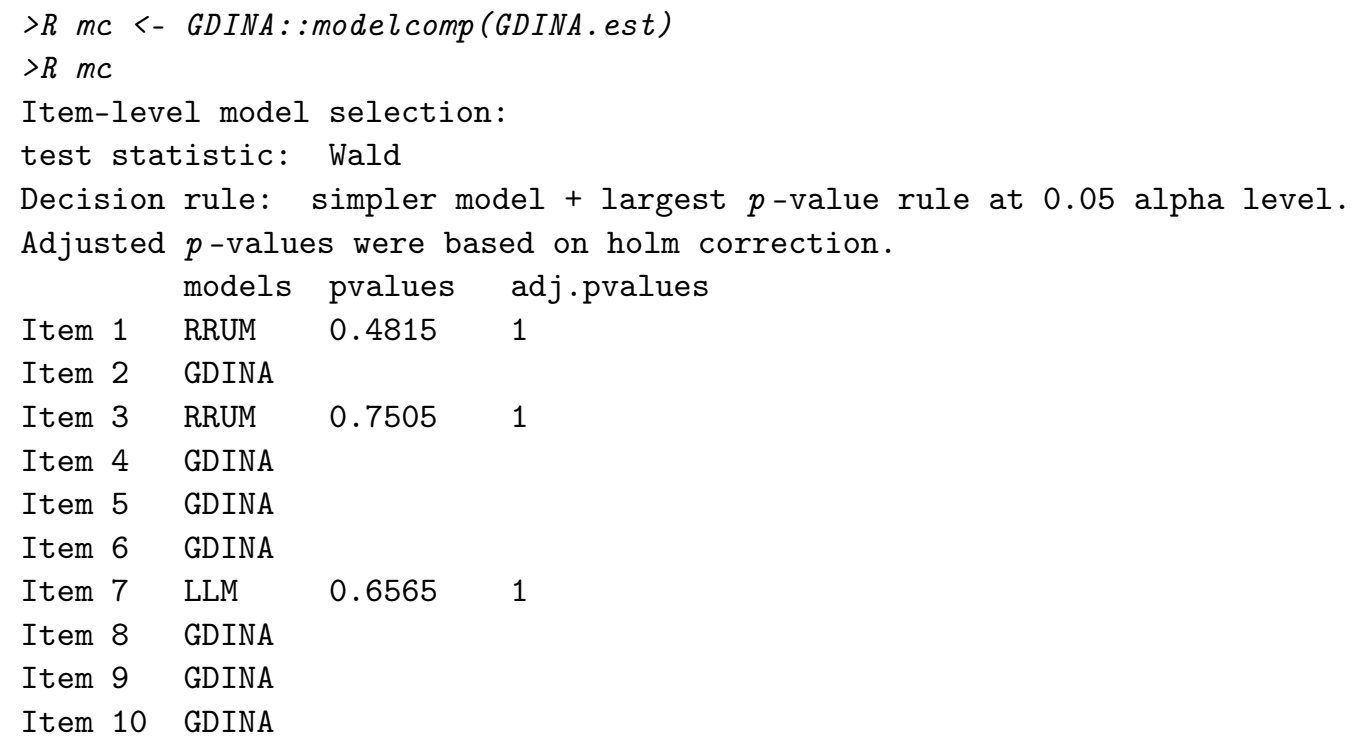




$\begin{array}{lllll}\text { Item } 11 & \text { ACDM } & 0.9209 & 1 \\ \text { Item } 12 & \text { RRUM } & 0.4902 & 1 \\ \text { Item } 13 & \text { GDINA } & & \\ \text { Item } 14 & \text { GDINA } & & \\ \text { Item } 15 & \text { GDINA } & & \\ \text { Item } 16 & \text { LLM } & 0.5678 & 1 \\ \text { Item } 17 & \text { DINO } & 0.1332 & 1 \\ \text { Item } 18 & \text { GDINA } & & \\ \text { Item } 19 & \text { GDINA } & & \\ \text { Item 20 } & \text { RRUM } & 0.3889 & 1 \\ \text { Item 21 } & \text { LLM } & 0.9537 & 1 \\ \text { Item 22 } & \text { GDINA } & & \\ \text { Item 23 } & \text { GDINA } & & \\ \text { Item 24 } & \text { GDINA } & & \\ \text { Item 25 } & \text { GDINA } & & \\ \text { Item } 26 & \text { GDINA } & & \\ \text { Item 27 } & \text { GDINA } & & \\ \text { Item 28 } & \text { GDINA } & & \\ \end{array}$

Similarly, the CDM package implements the Wald test for comparing the G-DINA model with the DINA model, the DINO model, A-CDM in CDM : : gdina . wald(). In addition, the CDM package allows researchers to fit the regularized G-DINA model using a variety of penalty terms. This is a flexible approach to simplifying the G-DINA model and interested readers may refer to Robitzsch [37] and Robitzsch and George [36] for more information. A caveat to the Wald and LR tests for model comparisons is that trivial discrepancy between two models may be detected when sample size is large and one should be aware that the logit link must be used when the regularized G-DINA model is specified in the CDM package.

\subsection{Model Fit Evaluation}

Both CDM and GDINA packages offer functions for assessing model-data fit. Table 2 shows the functions and the statistics calculated in each package. It is evident that both packages calculate various statistics for assessing both absolute and relative fit at test and item levels. This paper will not enumerate the outputs of all those statistics; instead, it will focus on absolute fit statistics, as only the G-DINA model was used, and present some results as an example.

\subsubsection{Test-Level Fit Evaluation}

Most test-level absolute fit measures gauge the discrepancy between observed quantities and model-implied counterparts. For example, the $\mathbf{M}_{2}$ statistic $[38,58,59]$ compares the univariate and bivariate distributions of observations and model predictions. Because it conforms to $\chi^{2}$ distribution, hypothesis tests can be conducted to assess whether the model fits data. However, it is well-known that a hypothesis test is affected by sample size, and a large sample may capture trivial discrepancies between the model and the data. To address this issue, the root mean square error of approximation $\left(\operatorname{RMSEA}_{2}\right)[39,60]$ and the standardized root mean square residual (SRMSR) $[39,61]$ can be used as effect-size measures. For both RMSEA 2 and SRMSR, a smaller value indicates a better absolute model data fit [62]. Simulation studies suggest that RMSEA2 < 0.03 indicates excellent fit, $0.03<$ RMSEA2 < 0.045 a good fit, and RMSEA2 $<0.045$ poor fit. SRMSR $<0.05$ indicates good model fit $[39,63]$. It should be noted that when the number of parameters is large, the $\mathrm{M}_{2}$ statistic, as well as RMSEA 2 , may not be calculable. 
Table 2. Model-data fit statistics.

\begin{tabular}{|c|c|c|c|c|c|}
\hline & & \multicolumn{2}{|c|}{ CDM } & \multicolumn{2}{|c|}{ GDINA } \\
\hline & & Function & Statistics & Function & Statistics \\
\hline \multirow{3}{*}{ Absolute fit } & Test-level & IRT.modelfit() & $\begin{array}{c}\max \left(X^{2}\right) \\
\text { MADcor } \\
\text { SRMSR } \\
\text { MADRESIDCOV } \\
\text { abs(fcor) }\end{array}$ & $\begin{array}{l}\text { modelfit() } \\
\text { itemfit() }\end{array}$ & $\begin{array}{c}\mathrm{M}_{2} \\
\text { RMSEA }_{2} \\
\text { SRMSR } \\
\text { MaxAD.r } \\
\text { MaxAD.LOR }\end{array}$ \\
\hline & Item-level & $\begin{array}{c}\text { IRT.RMSD() } \\
\text { itemfit.sx2() }\end{array}$ & $\begin{array}{c}\text { RMSD } \\
\text { RMSD_bc } \\
\text { MAD } \\
\text { MD } \\
\chi^{2} \\
S-\chi^{2} \\
\text { RMSEA }\end{array}$ & itemfit() & $\begin{array}{c}\text { MaxAD.r } \\
\text { MaxAD.LOR }\end{array}$ \\
\hline & Item-pair level & IRT.modelfit() & $\begin{array}{c}\chi^{2} \\
\text { fcor }\end{array}$ & itemfit() & $\begin{array}{c}\text { MaxAD.r } \\
\text { MaxAD.LOR }\end{array}$ \\
\hline \multirow[t]{2}{*}{ Relative fit } & Test-level & $\begin{array}{c}\text { IRT.modelfit() } \\
\text { anova() }\end{array}$ & $\begin{array}{c}\text { AIC } \\
\text { BIC } \\
\text { CAIC } \\
\text { AIC3 } \\
\text { AICc } \\
\text { LR test }\end{array}$ & $\begin{array}{l}\operatorname{modelfit}() \\
\text { anova() }\end{array}$ & $\begin{array}{c}\text { AIC } \\
\text { BIC } \\
\text { CAIC } \\
\text { SABIC } \\
\text { LR test }\end{array}$ \\
\hline & Item-level & gdina.wald() & Wald test & $\operatorname{model} \operatorname{comp}()$ & $\begin{array}{l}\text { Wald test } \\
\text { LR test }\end{array}$ \\
\hline
\end{tabular}

Note. $\max \left(\mathrm{X}^{2}\right)=$ the maximum chi-square statistic; MADcor = mean of absolute deviation of correlations; SRMSR = standardized mean square root of squared residuals; MADRESIDCOV = mean of absolute deviation of residual covariances; abs(fcor) = the absolute deviation of Fisher transformed correlations; RMSD = root mean square deviation; RMSD_bc = RMSD statistic with analytical bias correction; $\mathrm{MAD}=$ mean absolute deviation; $\mathrm{MD}=$ mean deviation; $\chi^{2}=$ chi-square statistic; $\mathrm{S}-\chi^{2}=\mathrm{S}$-chi-square statistic; $\mathrm{RMSEA}=$ the root mean square error of approximation; fcor $=$ Fisher transformed correlations; AIC = Akaike's Information Criteria; BIC = Bayesian Information Criteria; $\mathrm{CAIC}=$ consistent $\mathrm{AIC} ; \mathrm{AICc}=$ the sample size adjusted $\mathrm{AIC} ; \mathrm{M}_{2}=$ the second-order marginal statistic; $\mathrm{RMSEA}_{2}=$ limited information RMSEA; MaxAD. $r$ = maximum absolute deviation of transformed correlation; MaxAD.LOR = maximum absolute deviation of $\log$ odds ratio; $\mathrm{SABIC}=$ the sample size adjusted $\mathrm{BIC} ; \mathrm{LR}$ test $=$ likelihood ratio test.

Aggregated item-level or item-pair level absolute fit measures have also been used to assess test-level fit. Examples include the mean absolute difference between the observed and expected correlations (MADcor) [64,65], the maximum absolute difference between observed and predicted Fisher transformed correlations (MaxAD.r) [64], the maximum absolute difference between observed and predicted log odds ratios (MaxAD.LOR) [64], the mean of absolute deviations of residual covariances (MADRESIDCOV) [66], and the maximum $\chi^{2}$ value of all item pairs $\left(\max \left(\mathrm{X}^{2}\right)\right)$ [67]. The $\chi^{2}$ statistic quantifies the deviance between the observed and predicted item-pair distributions, using individual posterior distributions of the specified model. For MaxAD.r, MaxAD.LOR and $\max \left(X^{2}\right)$, one can often report adjusted $p$-value to assess whether the model and the data fit well for the worst pair of items. For other measures, a small value indicates a good fit. Since the value of MADRESIDCOV is often small, the value of $100^{*}$ MADRESIDCOV is usually adopted [68].

In the CDM package, function CDM: :IRT. modelfit () can be used to calculate MADcor, MaxAD.r (labelled as abs (fcor)), MADRESIDCOV, $\max \left(X^{2}\right)$ and SRMSR. The CDM: :IRT.modelfit () function also calculates information criteria, such as Akaike's Information Criteria (AIC) [69] and Bayesian Information Criteria (BIC) [70]. Both criteria are based on the maximum likelihood statistic, and BIC is additionally affected by sample size. Both AIC and BIC serve as a measure for comparing model fit, and a smaller value indicates better model fit. Nevertheless, please note that when parameters are fixed in model calibration, the calculation of information criteria is incorrect and must be manually corrected. 
In the GDINA package, GDINA : : model fit () function calculates $\mathrm{M}_{2}$ statistic, $\mathrm{RMSEA}_{2}$, and SRMSR for absolute fit evaluation, and calculates log-likelihood, AIC, BIC, CAIC, and SABIC for relative fit evaluation. The GDINA : : itemfit() function calculates MaxAD.r and MaxAD.LOR. The code below shows how to obtain these statistics from CDM and GDINA packages.

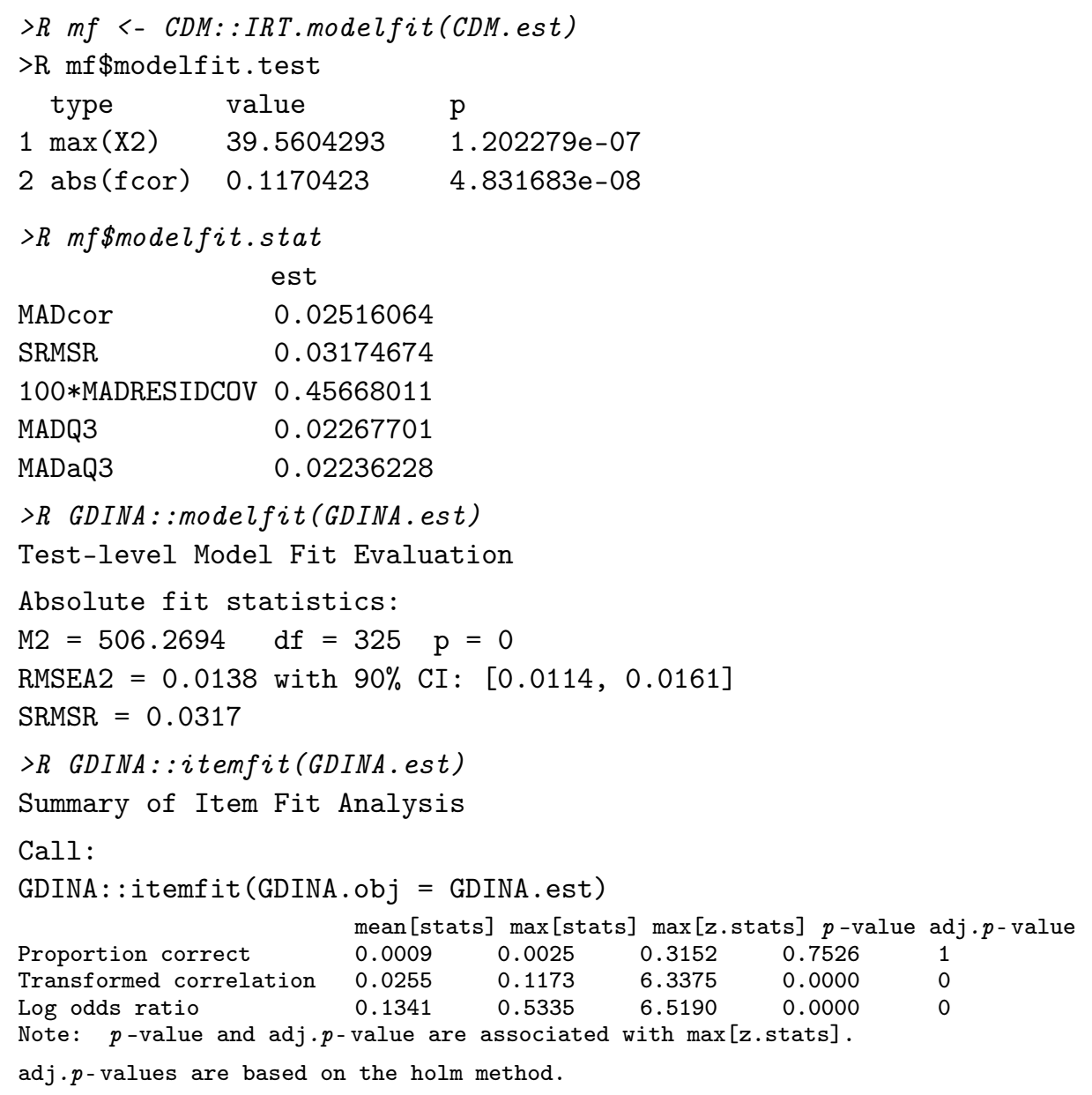

\subsubsection{Item-Level and Item-Pair Level Fit Evaluation}

Item-level absolute fit can be assessed using S- $\chi^{2}$ item fit statistic [41,71], which can be calculated using CDM: : itemfit.sx2() function. The S- $\chi^{2}$ item fit statistic compares observed and expected proportions for each item and each latent class and forms a chisquare distributed statistic. As a result, the items with $p$-values greater than 0.05 indicate good item fit at 0.05 nominal level. The output, for instance, indicates that item 13 has a significant misfit.

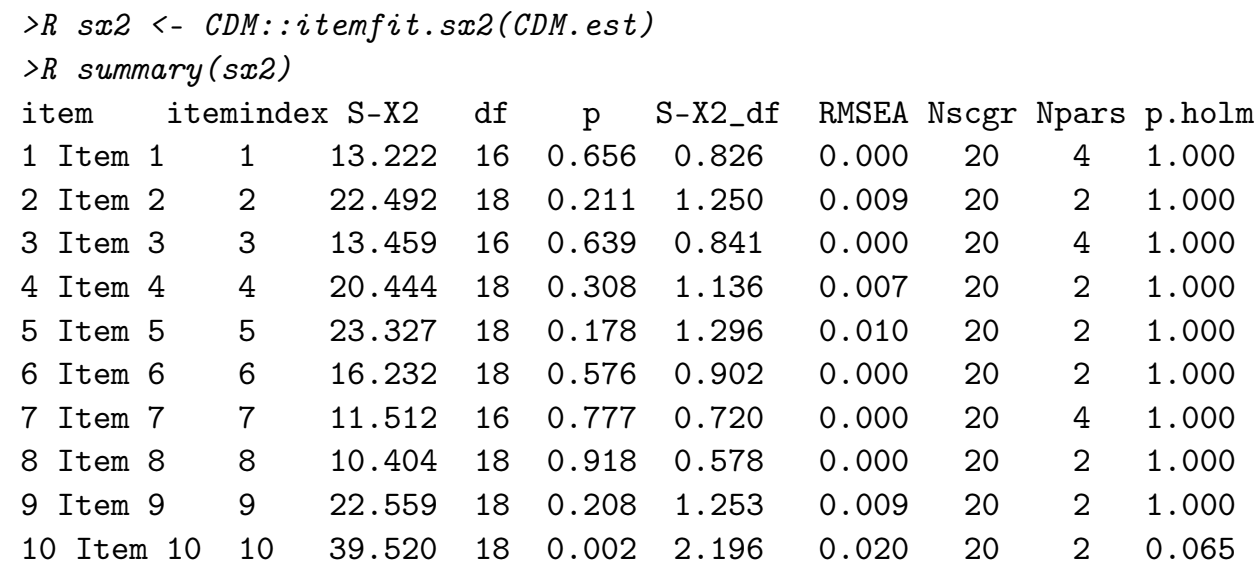




$\begin{array}{lllllllllll}11 & \text { Item } 11 & 11 & 18.609 & 16 & 0.289 & 1.163 & 0.007 & 20 & 4 & 1.000 \\ 12 & \text { Item } 12 & 12 & 20.022 & 16 & 0.219 & 1.251 & 0.009 & 20 & 4 & 1.000 \\ 13 & \text { Item 13 } & 13 & 55.254 & 18 & 0.000 & 3.070 & 0.027 & 20 & 2 & 0.000 \\ 14 & \text { Item } 14 & 14 & 18.053 & 18 & 0.452 & 1.003 & 0.001 & 20 & 2 & 1.000 \\ 15 & \text { Item 15 } & 15 & 15.154 & 18 & 0.651 & 0.842 & 0.000 & 20 & 2 & 1.000 \\ 16 & \text { Item 16 } & 16 & 32.079 & 16 & 0.010 & 2.005 & 0.019 & 20 & 4 & 0.254 \\ 17 & \text { Item 17 } & 17 & 13.134 & 16 & 0.663 & 0.821 & 0.000 & 20 & 4 & 1.000 \\ 18 & \text { Item 18 } & 18 & 17.355 & 18 & 0.499 & 0.964 & 0.000 & 20 & 2 & 1.000 \\ 19 & \text { Item 19 } & 19 & 33.059 & 18 & 0.016 & 1.837 & 0.017 & 20 & 2 & 0.410 \\ 20 & \text { Item 20 } & 20 & 14.929 & 16 & 0.530 & 0.933 & 0.000 & 20 & 4 & 1.000 \\ 21 & \text { Item 21 } & 21 & 15.607 & 16 & 0.481 & 0.975 & 0.000 & 20 & 4 & 1.000 \\ 22 & \text { Item 22 } & 22 & 27.171 & 18 & 0.076 & 1.509 & 0.013 & 20 & 2 & 1.000 \\ 23 & \text { Item 23 } & 23 & 16.777 & 18 & 0.538 & 0.932 & 0.000 & 20 & 2 & 1.000 \\ 24 & \text { Item 24 } & 24 & 18.182 & 18 & 0.444 & 1.010 & 0.002 & 20 & 2 & 1.000 \\ 25 & \text { Item 25 } & 25 & 16.269 & 18 & 0.574 & 0.904 & 0.000 & 20 & 2 & 1.000 \\ 26 & \text { Item 26 } & 26 & 25.935 & 18 & 0.101 & 1.441 & 0.012 & 20 & 2 & 1.000 \\ 27 & \text { Item 27 } & 27 & 24.753 & 18 & 0.132 & 1.375 & 0.011 & 20 & 2 & 1.000 \\ 28 & \text { Item 28 } & 28 & 13.008 & 18 & 0.791 & 0.723 & 0.000 & 20 & 2 & 1.000 \\ -- \text { Average Item Fit Statistics-- } & & & & & \\ \text { S-X2 = 21.019 I S-X2_df }=1.206 & & & & & \end{array}$

Other item-level absolute fit measures can be requested through function CDM : :IRT.RMSD () [63,72]. It computes item-wise and group-wise root mean square deviation (RMSD), bias corrected root mean square deviation (RMSD_bc), mean absolute deviation (MAD), mean deviation (MD), and chi square statistic $[73,74]$.

Unlike the S- $\chi^{2}$ and RMSD statistics that focus on to what extent the model can fit data well for each item, the absolute difference between observed and predicted Fisher transformed correlations and the absolute difference between observed and predicted log odds ratios for all item pairs [64] are reported in the GDINA package. Both measures focus on to what extent the model can explain the association between each pair of items. A heatmap plot illustrating the adjusted $p$-values of transformed correlation between item pairs can be requested using $p \operatorname{lot}($ ), as demonstrated in Figure 4 . In the heatmap plot, items are presented on both $\mathrm{x}$ - and $\mathrm{y}$-axes. The first item on the $x$-axis and the last on the $y$-axis were dropped for pairing items. The adjusted $p$-values of all item pairs are plotted in the lower right shading area, where those of adequately fitted item pairs are in grey $(p>0.05)$ and those of inadequately fitted item pairs are in different tones of red $(p<0.05)$, depending on the $p$-value [42]. In our case, some item pairs (e.g., items 9 and 10 and items 13 and 22) demonstrated significant misfit and thus are in demand for further exploration by domain experts.

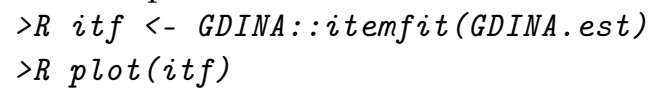




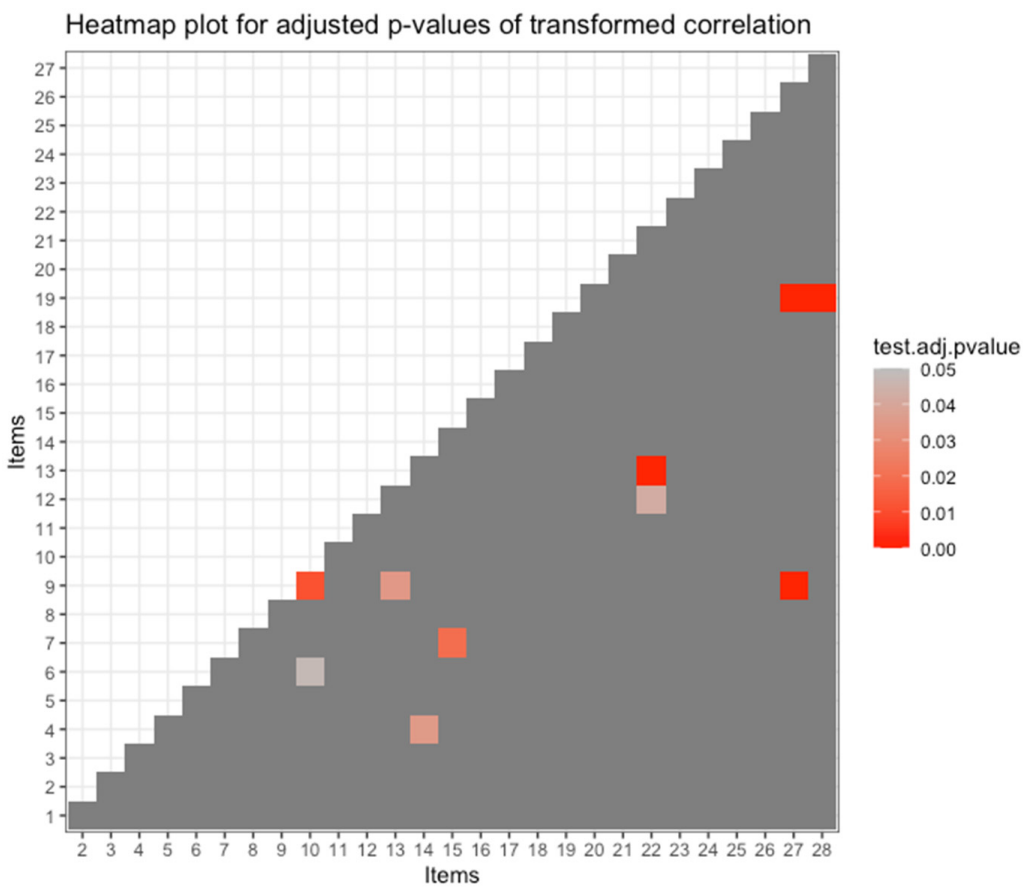

Figure 4. Heatmap plot for adjusted $p$-values for transformed correlation.

\subsection{Item Diagnosticity Investigation}

To assess item diagnosticity, the distribution of the probability of success across all latent groups in each item can be drawn, using plot() as the code presents below. The plots should show the distinctions between the bars representing each latent group. A good example is item 20 presented in Figure 5, where an increase is observed in the probability of success as a student masters more attributes measured by this item. A poor example is item 17, where the success probability of all four latent groups is over 0.75 and little difference is observed between the bars. This indicates that a student has a more than $75 \%$ chance to answer this item correctly whether they do not master any attributes required by this item, master only one attribute, or master both attributes. In this regard, item 17 does not have the ability to distinguish students in different latent groups. Similar plots can be drawn using the $p \operatorname{lot}($ ) function as well in the CDM package.
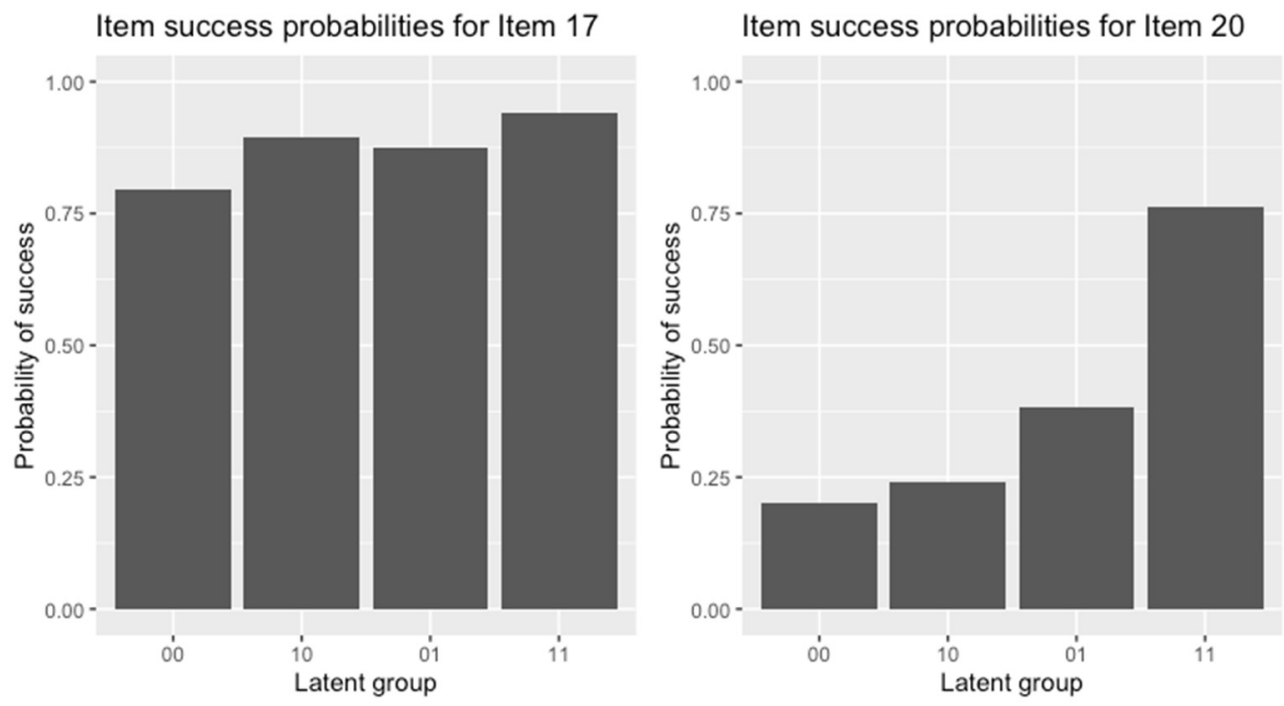

Figure 5. Plots of Success Probabilities of Items 17 and 20. 
$>R$ plot(GDINA.est, item $=1: 28$ )

Another way to check item diagnosticity is to investigate the item discrimination indices. In the GDINA package, item discrimination is measured by two indices: $\mathrm{P}(1)-\mathrm{P}(0)$ and G-DINA discrimination index (GDI). $\mathrm{P}(1)-\mathrm{P}(0)$ measures the differences in success probabilities between those who master all required attributes and those who master none of them. GDI measures the variance of the item success probabilities based on the reduced attribute profile $[28,75]$. An item with a higher value of $\mathrm{P}(1)-\mathrm{P}(0)$ or GDI has higher discrimination power. Currently, there is no agreement in the field regarding the value of good discrimination power. Although a higher value of $\mathrm{P}(1)-\mathrm{P}(0)$ or GDI is desirable, it could be an indicator of overspecified q-vectors [28]. These two item discrimination indices can be requested using GDINA : : extract () as in:

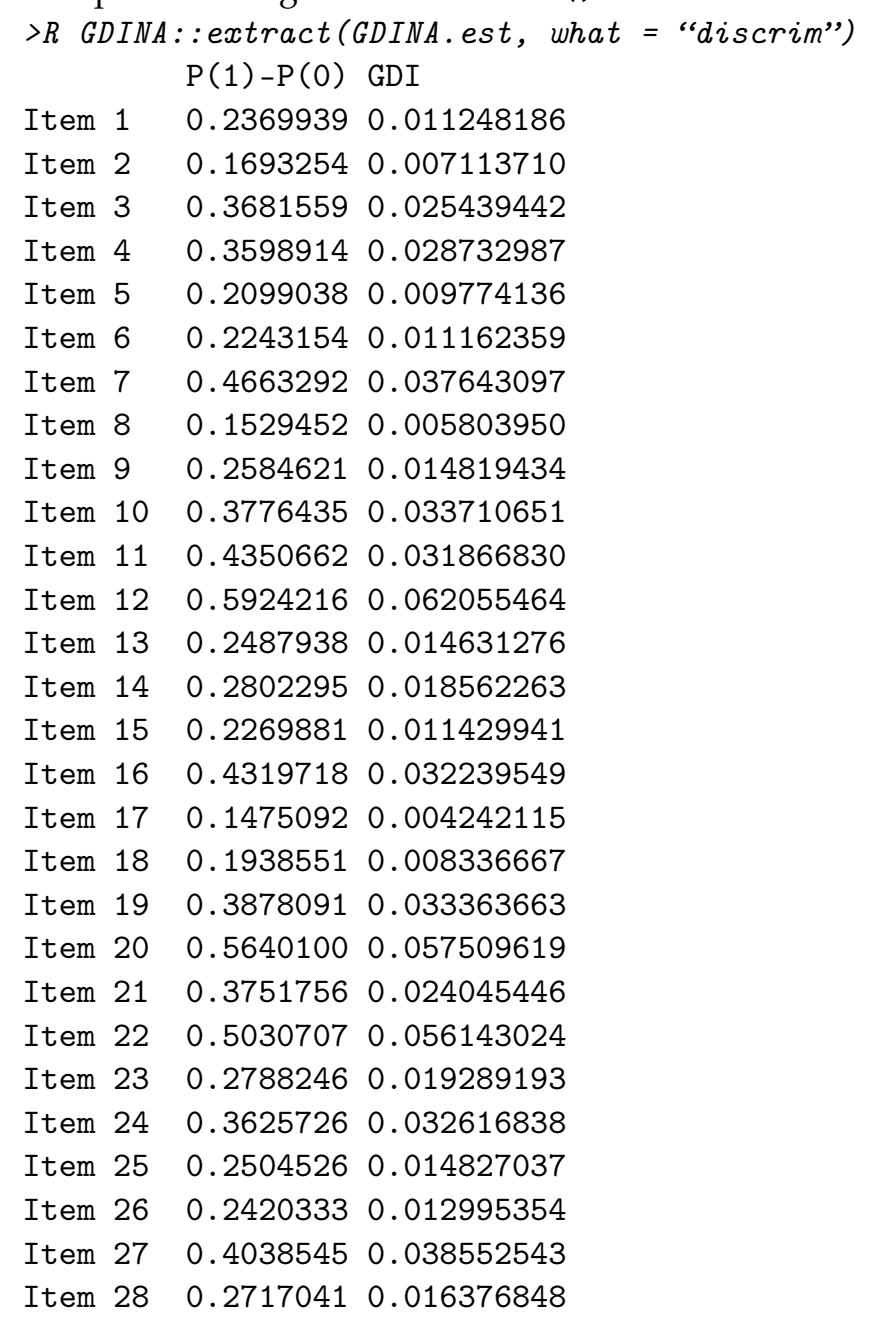

In the CDM package, $\mathrm{P}(1)-\mathrm{P}(0)$ is referred to as item discrimination index or IDI $[48,76]$. The CDM package also calculates the discrimination index (DI) at the item-attribute level based on the mastery probability of including and excluding the measured attribute for a specific item and the DI at the test level by averaging the marginalized probability of DIs at the item-attribute level for each item. Using CDM: :discrim.index(), the test, item, and item-attribute level DIs can be requested. Note that the IDI at item level is the same as the $\mathrm{P}(1)-\mathrm{P}(0)$ values requested by GDINA : : extract (). Although not presented here, the CDM package also calculates the cognitive diagnostic index (CDI) based on the Kullback-Leibler information (KLI) [76], which can be requested by using CDM: :cdi.kli().

$>R$ summary (CDM: :discrim.index(CDM.est))

CDM 7.5-15 (2020-03-10 14:19:21) 
Test-level discrimination index

[1] 0.304

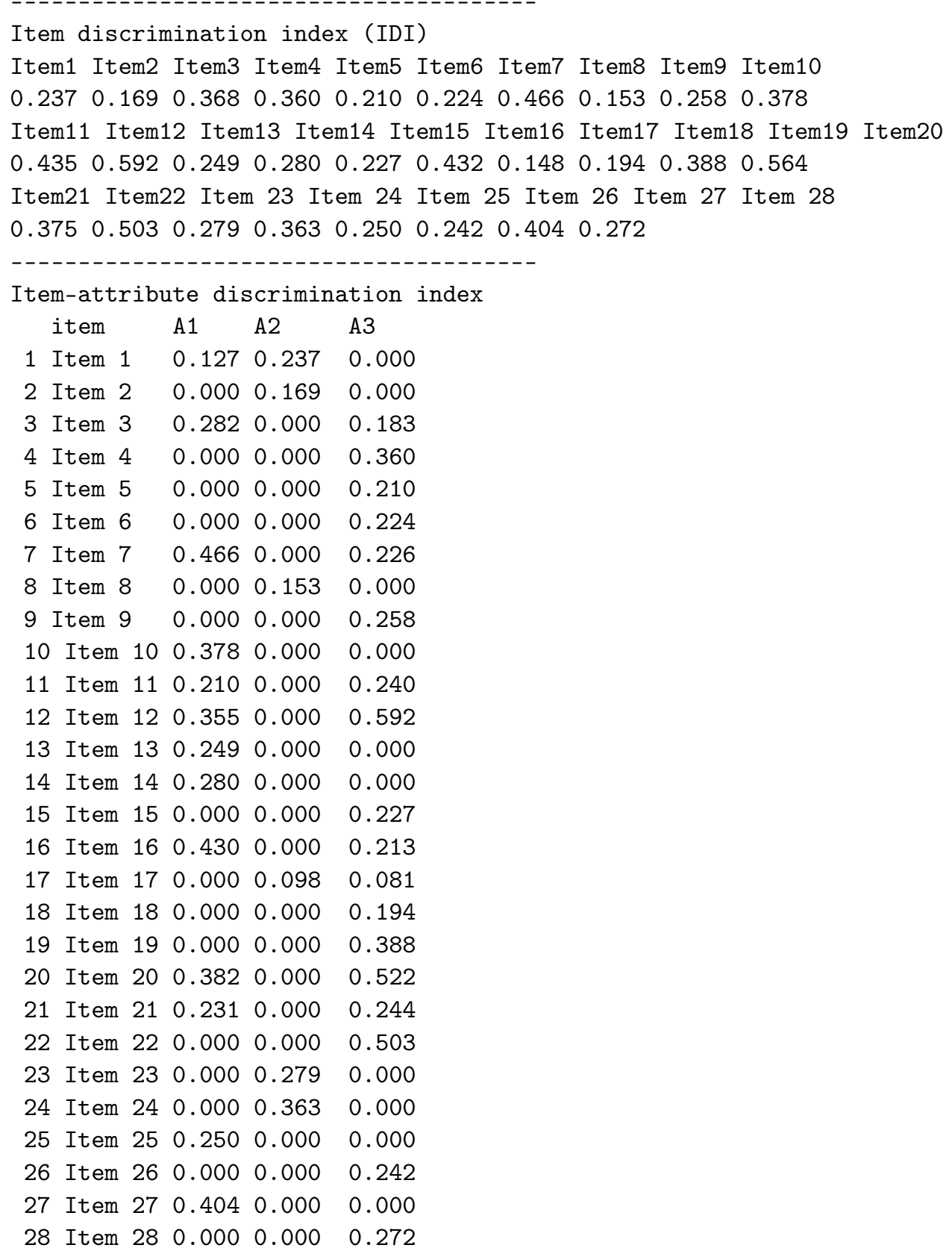

\subsection{Classification Reliability}

Classification reliability refers to whether the model can consistently and accurately classify test-takers into latent classes, usually measured by classification accuracy and consistency. Specifically, classification accuracy relates to the extent to which the estimated attribute classifications and the true classifications are the same, whereas classification consistency concerns the extent to which the estimated attribute classifications from two parallel test forms are consistent. Although different measures have been proposed in the literature [77-79], the GDINA package calculates classification accuracy at test, pattern, and attribute levels according to Iaconangelo [80] and Wang et al. [81]. In contrast, the CDM package calculates both classification accuracy and consistency at pattern and attribute levels using the estimator of Johnson and Sinharay [78]. 
The classification accuracy of maximum a posteriori or MAP method (by default, and maximum likelihood estimation or MLE can be requested) can be estimated in the GDINA package using GDINA : :CA() as demonstrated below:

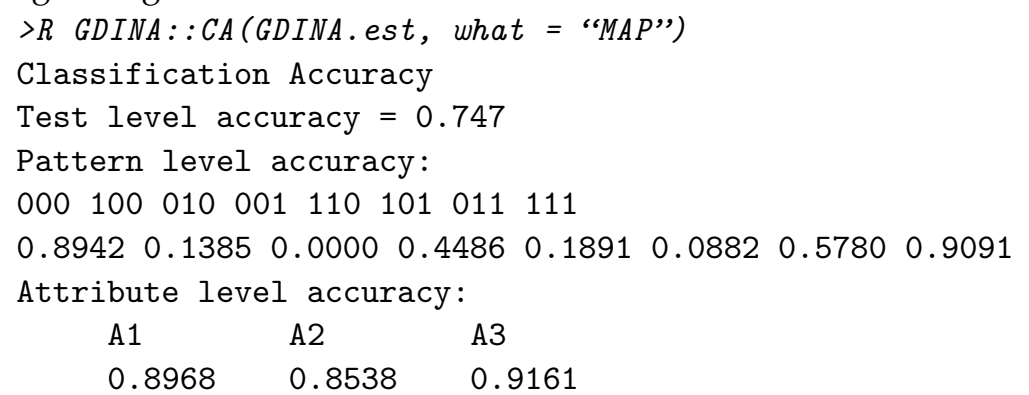

In the CDM package, one can calculate both classification accuracy and consistency using CDM: :cdm.est.class.accuracy() as demonstrated below. The output gives classification accuracy and consistency statistics [77,81] at attribute and latent class level for both MLE and MAP estimators. Pa_est and Pc_est give classification accuracy and consistency by estimators of Johnson and Sinharay [78,82], respectively, and Pa_sim and Pc_sim give classification accuracy and consistency only for DINA, DINO, and mixed DINA and DINO models based on simulation, respectively. The classification accuracy values at latent class and attribute level for MAP estimators are the same as the values we obtained from the GDINA package.

\begin{tabular}{|c|c|c|c|c|}
\hline & Pa_est & Pa_sim & Pc_est & Pc_sim \\
\hline MLE_patt & 0.594 & 0.621 & 0.409 & 0.437 \\
\hline MAP_patt & 0.747 & 0.771 & 0.664 & 0.686 \\
\hline MLE_A1 & 0.859 & 0.886 & 0.760 & 0.798 \\
\hline MLE_A2 & 0.759 & 0.762 & 0.645 & 0.645 \\
\hline MLE_A3 & 0.893 & 0.911 & 0.811 & 0.838 \\
\hline MAP_A1 & 0.896 & 0.913 & 0.833 & 0.849 \\
\hline MAP_A2 & 0.854 & 0.860 & 0.807 & 0.817 \\
\hline MAP_A3 & 0.916 & 0.933 & 0.854 & 0.882 \\
\hline
\end{tabular}

\subsection{CDM Result Presentation}

The primary goal of CDM analysis is to classify students into different latent classes or estimate students' attribute profiles. Both CDM and GDINA packages can estimate a person's parameters using expected a posteriori (EAP), MAP, or MLE methods [83]. In the GDINA package, the GDINA : : personparm() function could be used, whereas in the CDM package, the CDM: :IRT. factor.scores() function can be applied. Below are the estimated attribute profiles of the first six students using GDINA : : personparm().

$>R$ head(personparm(GDINA.est))

$\begin{array}{llll} & \text { A1 } & \text { A2 } & \text { A3 } \\ {[1,]} & 1 & 1 & 1 \\ {[2,]} & 1 & 1 & 1 \\ {[3,]} & 1 & 1 & 1 \\ {[4,]} & 1 & 1 & 1 \\ {[5,]} & 1 & 1 & 1 \\ {[6,]} & 1 & 1 & 1\end{array}$

Meanwhile, we can obtain the success probability of attribute mastery for each student by specifying the what argument to "mp" in GDINA : :personparm() function.

$>R$ head(personparm(GDINA.est, what = "mp"))

A1 A2 A3

$\left[\begin{array}{lllll}1,] & 0.9967 & 0.9615 & 0.9999\end{array}\right.$

$[2] \quad 0.9952 \quad 0.9150 \quad$,

$[3] \quad 0.98410 .9898 \quad$, 
$[4] \quad 0.9976 \quad 0.9913 \quad$,

$\left[\begin{array}{lllll}5,] & 0.9884 & 0.9845 & 0.9512\end{array}\right.$

$[6] \quad$,

Using the mpRadar () function created from the fmsb: :radarchart () function of the fmsb R package [84], a radar chart of the mastery probability for each student or for several students at the same time can be plotted. As presented in Figure 6, the student has a nearly $100 \%$ chance of mastering the lexical rules, a nearly $0 \%$ chance of mastering the morphosyntactic rules, and about a $45 \%$ chance of mastering the cohesive rules.

\section{Morphosyntactic}

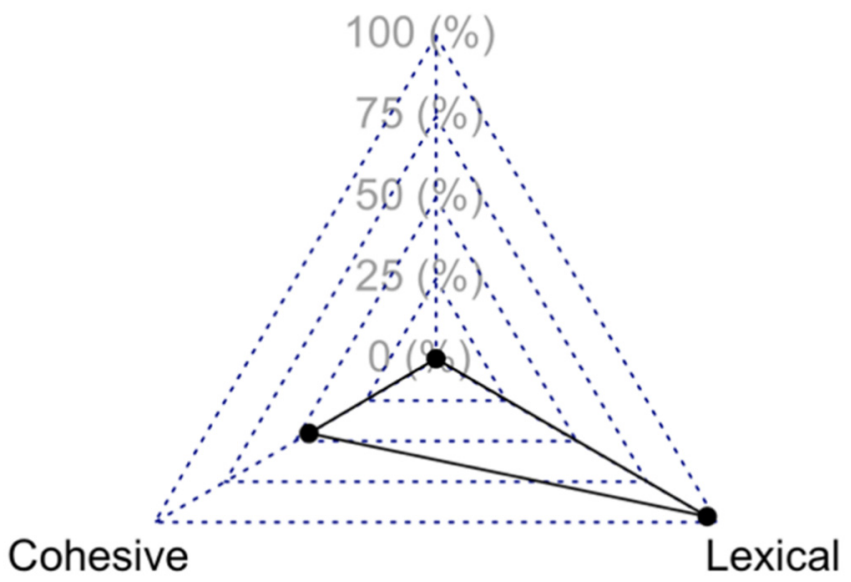

Figure 6. Radar Chart of the attribute mastery probabilities of student 8 .

In addition to person classifications, the proportion of students who master or do not master each attribute referred to as attribute prevalence, and the proportion of students in each latent class referred to as latent class proportions can also be measured. They can be requested by calling GDINA : : extract() function and specifying what = "prevalence" and "posterior.prob" in the GDINA package, respectively. In the CDM package, it can be obtained in the list named "Skill Pattern Probabilities" by calling the summary() function. Figure 7 presents a bar plot of the attribute prevalence, and Figure 8 presents a pie chart and a doughnut chart of the latent class proportions.

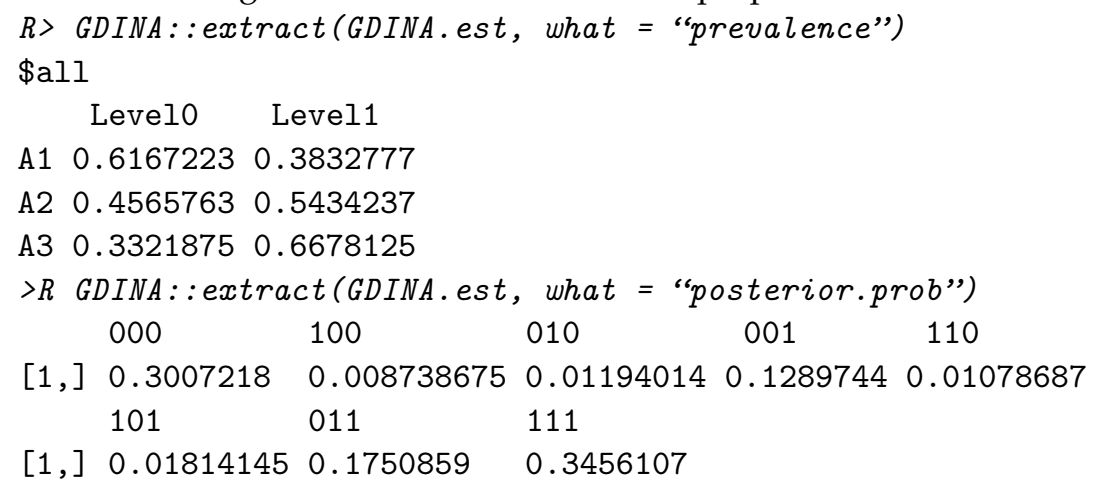

Figure 9 presents a network plot showing both the tetrachoric correlations among attributes and the attribute prevalence. In particular, the tetrachoric correlations are displayed on the arrows between corresponding attributes, and the attribute prevalence is represented using pie charts for each attribute. 


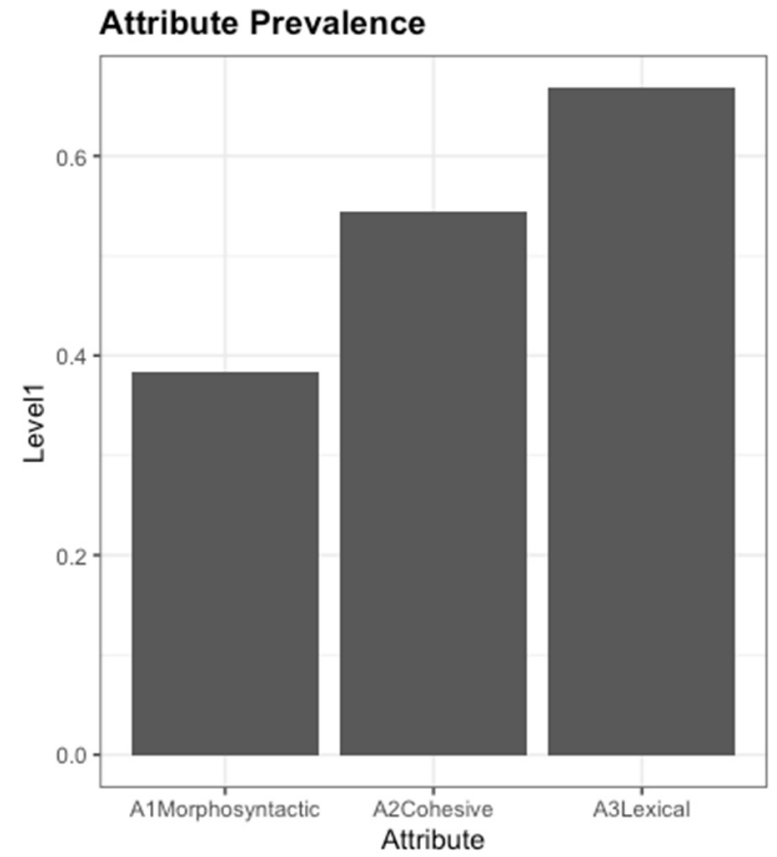

Figure 7. The bar plot of attribute prevalence.

\section{Latent Class Proportions}

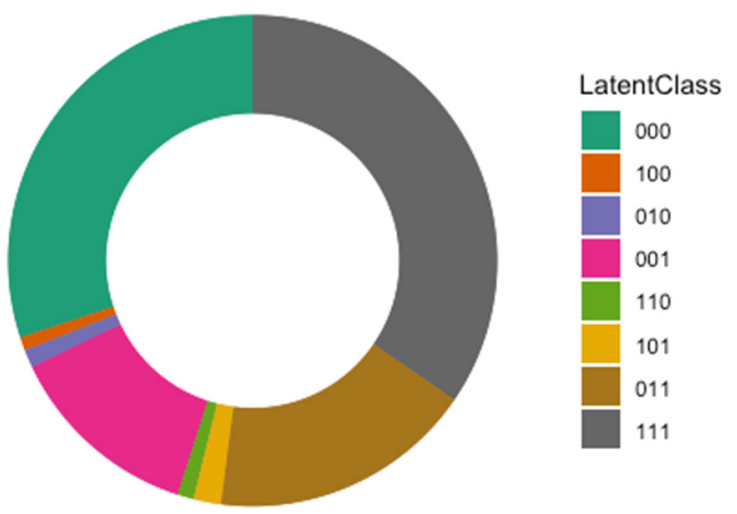

Figure 8. The doughnut chart of latent class proportions.

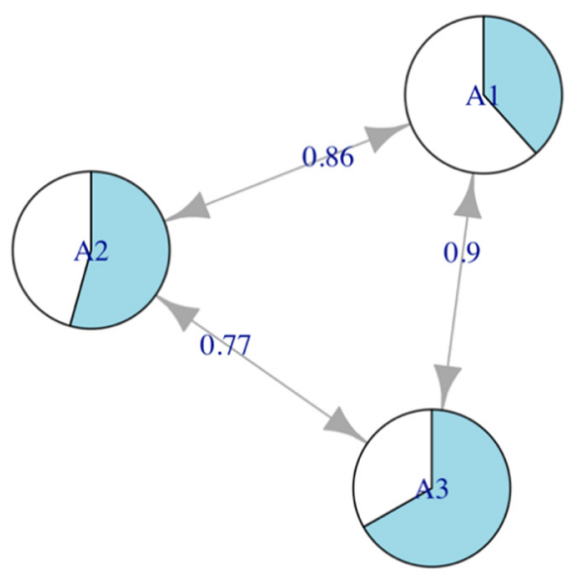

Figure 9. Network plot for attribute correlations and prevalence. 
The attribute prevalence and latent class proportions can be plotted together as presented in Figure 10, similar to Bradshaw et al. [2]. The code for creating the plots in Figures 7-10 was written by the authors and can be requested from the first author of the article.

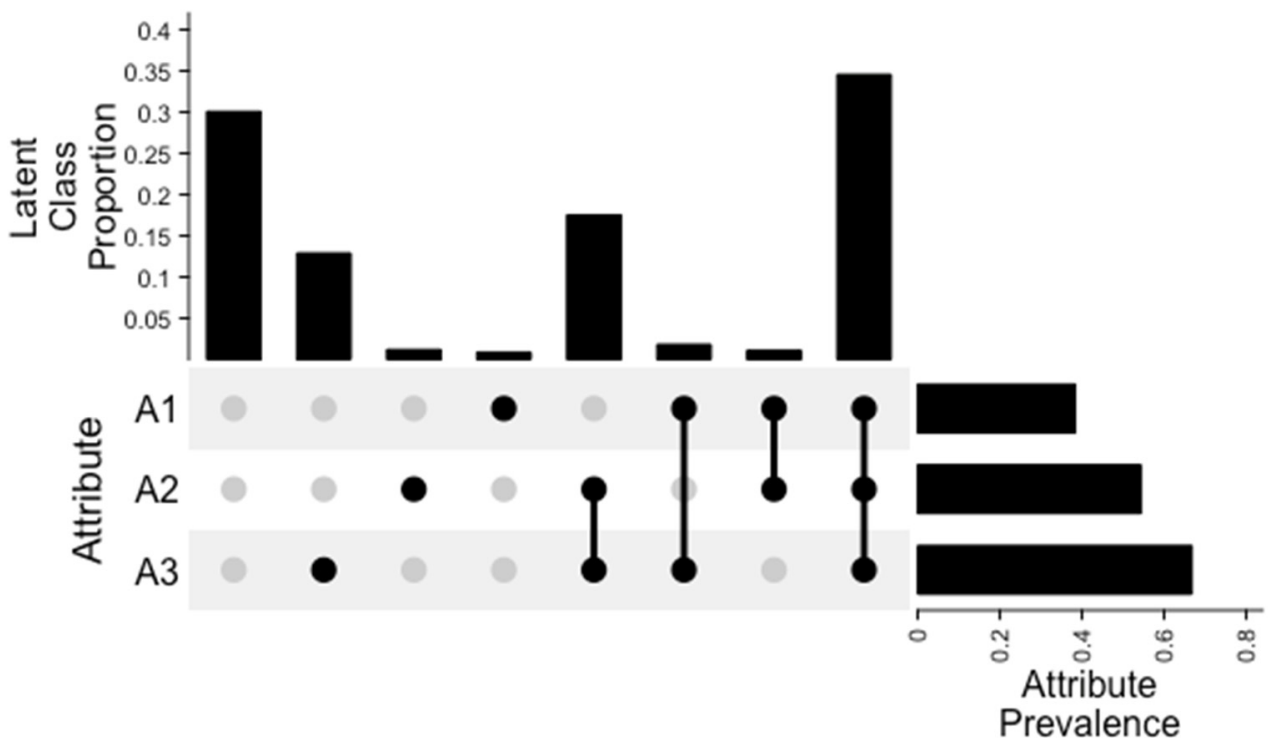

Figure 10. Attribute prevalence and latent class proportions.

\section{Discussion}

The purpose of this study is to provide a hands-on example of conducting CDM analysis in the G-DINA framework using $R$ packages and illustrating how different $R$ packages can be used in an integrated manner, providing richer information for cognitive diagnosis. Utilizing an exemplary dataset, the study demonstrated a workflow of CDM analyses, from Q-matrix validation to classification visualization. Such an illustration will be helpful to researchers who plan to conduct CDM analysis in R. However, only a limited number of relevant procedures were discussed because of their availability in existing $R$ packages. Other procedures that are equally if not more critical can often be found in the literature.

Despite the potential usefulness, the procedures discussed in this paper may not always work well. For example, the $\mathrm{M}_{2}$ statistic and RMSEA ${ }_{2}$ from the GDINA package may not be calculable if the number of parameters is too large. The S- $\chi^{2}$ item fit statistic from the CDM package would also not be calculated if there are missing data. In addition, although it was shown that the CDM and GDINA packages could complement each other in various aspects, researchers need to proceed with caution when using them together. Separate data calibrations may produce different parameter estimates due to the fact that (1) the EM algorithm may reach local maxima or (2) different default settings are specified for different packages. Therefore, this paper shows how to obtain equivalent calibration results by fixing parameter estimates obtained from one package in the other. Doing so, however, may lead to incorrect calculation of the number of free parameters and consequently affect the calculation of other statistics, such as information criteria.

Finally, it should be emphasized that this paper only focuses on the CDM analysis of dichotomous response data using the G-DINA model. However, researchers can do more than that in R. For example, the CDM package can also handle the general diagnostic model and regularized latent class model, while the GDINA package can handle several CDMs for multiple strategies. Both can also run CDMs for polytomous attributes and polytomous responses. Also, The NPCD and ACTCD [49,85] packages can conduct nonparametric cognitive diagnostic analysis. 
Author Contributions: Conceptualization, Q.S. and W.M.; methodology, Q.S., W.M., A.R. and M.A.S.; formal analysis, Q.S., W.M. and M.A.S.; writing—original draft preparation, Q.S. and W.M.; writingreview and editing, Q.S., W.M., A.R., M.A.S. and K.M.; visualization, Q.S. and W.M. All authors have read and agreed to the published version of the manuscript.

Funding: This research received no external funding.

Informed Consent Statement: Not applicable.

Data Availability Statement: Publicly available datasets were analyzed in this study. This data can be found here: [https: / CRAN.R-project.org/package=GDINA], accessed on 4 December 2021.

Conflicts of Interest: The authors declare no conflict of interest.

\section{References}

1. Ma, W.; Minchen, N.; de la Torre, J. Choosing between CDM and unidimensional IRT: The proportional reasoning test case. Measurement 2020, 18, 87-96. [CrossRef]

2. Bradshaw, L.; Izsák, A.; Templin, J.; Jacobson, E. Diagnosing teachers' understandings of rational numbers: Building a multidimensional test within the diagnostic classification framework. Educ. Meas. 2014, 33, 2-14. [CrossRef]

3. Wang, S.; Yang, Y.; Culpepper, S.A.; Douglas, J.A. Tracking skill acquisition with cognitive diagnosis models: A higher-order, hidden Markov model with covariates. J. Educ. Behav. Stat. 2018, 43, 57-87. [CrossRef]

4. George, A.C.; Robitzsch, A. Validating theoretical assumptions about reading with cognitive diagnosis models. Int. J. Test. 2021, 21, 105-129. [CrossRef]

5. Sorrel, M.A.; Olea, J.; Abad, F.J.; de la Torre, J.; Aguado, D.; Lievens, F. Validity and reliability of Situational Judgement Test scores: A new approach based on cognitive diagnosis models. Organ. Res. Methods 2016, 19, 506-532. [CrossRef]

6. Templin, J.L.; Henson, R.A. Measurement of psychological disorders using cognitive diagnosis models. Psychol. Methods 2006, 11, 287-305. [CrossRef]

7. De la Torre, J.; van der Ark, L.A.; Rossi, G. Analysis of clinical data from a cognitive diagnosis modeling framework. Meas. Eval. Couns. Dev. 2018, 51, 281-296. [CrossRef]

8. Muthén, L.; Muthén, B. Mplus. Version 8; Muthén \& Muthén: Los Angeles, CA, USA, 1998-2017.

9. Templin, J.; Hoffman, L. Obtaining diagnostic classification model estimates using Mplus. Educ. Meas. 2013, 32, 37-50. [CrossRef]

10. Plummer, M. JAGS: A program for analysis of Bayesian graphical models using Gibbs sampling. In Proceedings of the 3rd International Workshop on Distributed Statistical Computing, Vienna, Austria, 20-22 March 2003; p. 124.

11. Zhan, P.; Jiao, H.; Man, K.; Wang, L. Using JAGS for Bayesian cognitive diagnosis modeling: A tutorial. J. Educ. Behav. Stat. 2019, 44, 473-503. [CrossRef]

12. Carpenter, B.; Gelman, A.; Hoffman, M.D.; Lee, D.; Goodrich, B.; Betancourt, M.; Brubaker, M.A.; Guo, J.; Li, P.; Riddell, A. Stan: A probabilistic programming language. J. Stat. Soft. 2017, 76, 1-32. [CrossRef]

13. Jiang, Z.; Carter, R. Using Hamiltonian Monte Carlo to estimate the log-linear cognitive diagnosis model via Stan. Behav. Res. Methods 2019, 51, 651-662. [CrossRef] [PubMed]

14. George, A.C.; Robitzsch, A.; Kiefer, T.; Groß, J.; Ünlü, A. The R package CDM for cognitive diagnosis models. J. Stat. Soft. 2016, 74, 1-24. [CrossRef]

15. Ma, W.; de la Torre, J. GDINA: An R package for cognitive diagnosis modeling. J. Stat. Soft. 2020, 93, 1-26. [CrossRef]

16. De la Torre, J. The generalized DINA model framework. Psychometrika 2011, 76, 179-199. [CrossRef]

17. Nichols, P.D.; Chipman, S.F.; Brennan, R.L. (Eds.) Cognitively Diagnostic Assessment; Erlbaum: Hills-dale, NJ, USA, 1995.

18. Tjoe, H.; de la Torre, J. The identification and validation process of proportional reasoning attributes: An application of a cognitive diagnosis modeling framework. Math. Ed. Res. J. 2014, 26, 237-255. [CrossRef]

19. Von Davier, M. A general diagnostic model applied to language testing data. Br. J. Math. Stat. Psychol. 2008, 61, 287-307. [CrossRef] [PubMed]

20. Haertel, E.H. Using restricted latent class models to map the skill structure of achievement items. J. Educ. Meas. 1989, 26, 301-321. [CrossRef]

21. Junker, B.W.; Sijtsma, K. Cognitive assessment models with few assumptions, and connections with nonparametric Item Response Theory. Appl. Psychol. Meas. 2001, 25, 258-272. [CrossRef]

22. De la Torre, J.; Douglas, J.A. Higher-order latent trait models for cognitive diagnosis. Psychometrika 2004, 69, 333-353. [CrossRef]

23. Maris, E. Estimating multiple classification latent class models. Psychometrika 1999, 64, 187-212. [CrossRef]

24. Hartz, S.M. A Bayesian Framework for the Unified Model for Assessing Cognitive Abilities: Blending Theory with Practicality. Diss. Abstr. Int. B Sci. Eng. 2002, 63, 864.

25. Leighton, J.P.; Gierl, M.J. Cognitive Diagnostic Assessment for Education: Theory and Applications; Cambridge University Press: Cambridge, UK, 2007.

26. Henson, R.A.; Templin, J.L.; Willse, J.T. Defining a family of cognitive diagnosis models using log-linear models with latent variables. Psychometrika 2009, 74, 191-210. [CrossRef] 
27. Nájera, P.; Abad, F.J.; Sorrel, M.A. Determining the number of attributes in cognitive diagnosis modeling. Front. Psychol. 2021, 12, 321. [CrossRef]

28. De la Torre, J.; Chiu, C.-Y. A general method of empirical Q-matrix validation. Psychometrika 2016, 81, 253-273. [CrossRef] [PubMed]

29. Ma, W.; de la Torre, J. An empirical Q-matrix validation method for the sequential generalized DINA model. Br. J. Math. Stat. Psychol. 2020, 73, 142-163. [CrossRef] [PubMed]

30. Nájera, P.; Sorrel, M.A.; Abad, F.J. Reconsidering cutoff points in the general method of empirical Q-Matrix validation. Educ. Psychol. Meas. 2019, 79, 727-753. [CrossRef]

31. Nájera, P.; Sorrel, M.A.; de la Torre, J.; Abad, F.J. Improving robustness in Q-Matrix validation using an iterative and dynamic procedure. Appl. Psychol. Meas. 2020, 44, 431-446. [CrossRef]

32. De la Torre, J.; Minchen, N.D. The G-DINA model framework. In Handbook of Diagnostic Classification Models; von Davier, M., Lee, Y.-S., Eds.; Springer International Publishing: Cham, Switzerland, 2019; pp. 155-169.

33. De la Torre, J.; Lee, Y.-S. Evaluating the Wald Test for item-level comparison of saturated and reduced models in cognitive diagnosis. J. Educ. Meas. 2013, 50, 355-373. [CrossRef]

34. Ma, W.; Iaconangelo, C.; de la Torre, J. Model similarity, model selection, and attribute classification. Appl. Psychol. Meas. 2016, 40, 200-217. [CrossRef]

35. Sorrel, M.A.; de la Torre, J.; Abad, F.J.; Olea, J. Two-step likelihood ratio test for item-level model comparison in cognitive diagnosis models. Methodology 2017, 13, 39-47. [CrossRef]

36. Robitzsch, A.; George, A.C. The R package CDM for diagnostic modeling. In Handbook of Diagnostic Classification Models; von Davier, M., Lee, Y.-S., Eds.; Springer International Publishing: Cham, Switzerland, 2019; pp. 549-572.

37. Robitzsch, A. Regularized latent class analysis for polytomous item responses: An application to SPM-LS data. J. Intell. 2020, 8, 30. [CrossRef]

38. Ma, W.; Jiang, Z. Estimating cognitive diagnosis models in small samples: Bayes modal estimation and monotonic constraints. Appl. Psychol. Meas. 2021, 45, 95-111. [CrossRef]

39. Maydeu-Olivares, A.; Joe, H. Assessing approximate fit in categorical data analysis. Multivariate Behav. Res. 2014, 49, 305-328. [CrossRef] [PubMed]

40. Liu, Y.; Tian, W.; Xin, T. An application of $\mathrm{M}_{2}$ statistic to evaluate the fit of cognitive diagnostic models. J. Educ. Behav. Stat. 2016, 41, 3-26. [CrossRef]

41. Sorrel, M.A.; Abad, F.J.; Olea, J.; de la Torre, J.; Barrada, J.R. Inferential item-fit evaluation in cognitive diagnosis modeling. Appl. Psychol. Meas. 2017, 41, 614-631. [CrossRef]

42. Ma, W. Cognitive diagnosis modeling using the GDINA R package. In Handbook of Diagnostic Classification Models; von Davier, M., Lee, Y.-S., Eds.; Springer International Publishing: Cham, Switzerland, 2019; pp. 593-601.

43. Balamuta, J.J.; Culpepper, S.A.; Douglas, J.A. edmdata: Data Sets for Psychometric Modeling; R Package Version 1.2.0; 2021. Available online: https:/ /CRAN.R-project.org/package=edmdata (accessed on 9 September 2021).

44. Nájera, P.; Sorrel, M.A.; Abad, F.J. cdmTools: Useful Tools for Cognitive Diagnosis Modeling; R Package Version 1.0.0. 2021. Available online: https:/ /CRAN.R-project.org/package=cdmTools (accessed on 14 September 2021).

45. Garrido, L.E.; Abad, F.J.; Ponsoda, V. A new look at Horn's parallel analysis with ordinal variables. Psychol. Methods 2013, 18, 454-474. [CrossRef] [PubMed]

46. Wang, W.; Song, L.; Ding, S. An exploratory discrete factor loading method for Q-Matrix specification in cognitive diagnostic models. In Springer Proceedings in Mathematics \& Statistics, Quantitative Psychology, IMPS, 2017; Wiberg, M., Culpepper, S., Janssen, R., González, J., Molenaar, D., Eds.; Springer: Cham, Switzerland, 2018; Volume 233. [CrossRef]

47. Nájera, P.; Sorrel, M.A.; de la Torre, J.; Abad, F.J. Balancing fit and parsimony to improve Q-matrix validation. Br. J. Math. Stat. Psychol. 2021, 74, 110-130. [CrossRef]

48. De la Torre, J. An empirically based method of Q-matrix validation for the DINA model: Development and applications. J. Educ. Meas. 2008, 45, 343-362. [CrossRef]

49. Zheng, Y.; Chiu, C.-Y. NPCD: Nonparametric Methods for Cognitive Diagnosis; R Package Version 1.0-11. 2019. Available online: https:/ /CRAN.R-project.org/package=NPCD (accessed on 9 September 2021).

50. Chiu, C.-Y. Statistical refinement of the Q-matrix in cognitive diagnosis. Appl. Psychol. Meas. 2013, 37, 598-618. [CrossRef]

51. De la Torre, J.; Ma, W. Cognitive diagnosis modeling: A general framework approach and its implementation in R. In Proceedings of the Fourth Conference on Statistical Methods in Psychometrics, Columbia University, New York, NY, USA, 30 August 2016.

52. Liu, J.; Xu, G.; Ying, Z. Data-driven learning of Q-matrix. Appl. Psychol. Meas. 2012, 36, 548-564. [CrossRef]

53. Chen, Y.; Liu, J.; Xu, G.; Ying, Z. Statistical analysis of Q-matrix based diagnostic classification models. J. Am. Stat. Assoc. 2015, 110, 850-866. [CrossRef] [PubMed]

54. Chen, Y.; Culpepper, S.A.; Chen, Y.; Douglas, J. Bayesian estimation of the DINA Q. Psychometrika 2018, 83, 89-108. [CrossRef]

55. Xu, G.; Shang, Z. Identifying latent structures in restricted latent class models. J. Am. Stat. Assoc. 2018, 113, 1284-1295. [CrossRef]

56. Xu, X.; von Davier, M. Fitting the Structured General Diagnostic Model to NAEP Data (RR-08-27); ETS Research Report Series; ETS: Princeton, NJ, USA, 2008.

57. Zhang, C.-H. Nearly unbiased variable selection under minimax concave penalty. Ann. Stat. 2010, 38, 894-942. [CrossRef] 
58. Maydeu-Olivares, A.; Joe, H. Limited information goodness-of-fit testing in multidimensional contingency tables. Psychometrika 2006, 71, 713-732. [CrossRef]

59. Hansen, M.; Cai, L.; Monroe, S.; Li, Z. Limited-information goodness-of-fit testing of diagnostic classification item response models. Br. J. Math. Stat. Psychol. 2016, 69, 225-252. [CrossRef] [PubMed]

60. Hu, J.; Miller, M.D.; Huggins-Manley, A.C.; Chen, Y.-H. Evaluation of model fit in cognitive diagnosis models. Int. J. Test. 2016, 16, 119-141. [CrossRef]

61. Maydeu-Olivares, A. Goodness-of-fit assessment of item response theory models. Measurement 2013, 11, 71-137. [CrossRef]

62. Ma, W. Evaluating the fit of sequential G-DINA model using limited-information measures. Appl. Psychol. Meas. 2020, 44, 167-181. [CrossRef]

63. Liu, R.; Huggins-Manley, A.C.; Bulut, O. Retrofitting diagnostic classification models to responses from IRT-based assessment forms. Educ. Psychol. Meas. 2018, 78, 357-383. [CrossRef]

64. Chen, J.; de la Torre, J.; Zhang, Z. Relative and absolute fit evaluation in cognitive diagnosis modeling. J. Educ. Meas. 2013, 50, 123-140. [CrossRef]

65. DiBello, L.V.; Roussos, L.A.; Stout, W.F. Review of cognitively diagnostic assessment and a summary of psychometric models. In Handbook of Statistics; Rao, C.R., Sinharay, S., Eds.; Elsevier: Amsterdam, The Netherlands, 2007; pp. 979-1030.

66. McDonald, R.P.; Mok, M.M.-C. Goodness of fit in item response models. Multivariate Behav. Res. 1995, 30, 23-40. [CrossRef] [PubMed]

67. Chen, W.; Thissen, D. Local dependence indexes for item pairs using item response theory. J. Educ. Behav. Stat. 1997, 22, 265-289. [CrossRef]

68. Xue, Z.; Juntao, W. On the sequential hierarchical cognitive diagnostic model. Front. Psychol. 2020, 11, 2562.

69. Akaike, H. A new look at the statistical identification model. IEEE Trans. Automat. Contr. 1974, 19, 716-723. [CrossRef]

70. Schwarz, G. Estimating the dimension of a model. Ann. Stat. 1978, 6, 461-464. [CrossRef]

71. Orlando, M.; Thissen, D. Likelihood-based item-fit indices for dichotomous item response theory models. Appl. Psychol. Meas. 2000, 24, 50-64. [CrossRef]

72. Kunina-Habenicht, O.; Rupp, A.A.; Wilhelm, O. A practical illustration of multidimensional diagnostic skills profiling: Comparing results from confirmatory factor analysis and diagnostic classification models. Stud. Educ. Eval. 2009, 35, 64-70. [CrossRef]

73. Oliveri, M.E.; von Davier, M. Investigation of model fit and score scale comparability in international assessments. Psychol. Test Assess. Model. 2011, 53, 315-333.

74. Yamamoto, K.; Khorramdel, L.; von Davier, M. Scaling PIAAC cognitive data. In Technical Report of the Survey of Adults Skills (PIAAC); Organisation for Economic Co-operation and Development(OECD), Ed.; OECD: Paris, France, 2013.

75. Kaplan, M.; de la Torre, J.; Barrada, J.R. New item selection methods for cognitive diagnosis computerized adaptive testing. Appl. Psychol. Meas. 2015, 39, 167-188. [CrossRef]

76. Henson, R.; DiBello, L.; Stout, B. A generalized approach to defining item discrimination for DCMs. Measurement 2018, 16, 18-29. [CrossRef]

77. Cui, Y.; Gierl, M.J.; Chang, H.-H. Estimating classification consistency and accuracy for cognitive diagnostic assessment. J. Educ. Meas. 2012, 49, 19-38. [CrossRef]

78. Johnson, M.S.; Sinharay, S. Measures of agreement to assess attribute-level classification accuracy and consistency for cognitive diagnostic assessments. J. Educ. Meas. 2018, 45, 635-664. [CrossRef]

79. Chen, Y.; Liu, Y.; Xu, S. Mutual information reliability for latent class analysis. Appl. Psychol. Meas. 2018, 42, 460-477. [CrossRef] [PubMed]

80. Iaconangelo, C. Uses of Classification Error Probabilities in the Three-Step Approach to Estimating Cognitive Diagnosis Models Doctoral Dissertation, Rutgers University, New Brunswick, NJ, USA, 2017, unpublished. [CrossRef]

81. Wang, W.; Song, L.; Chen, P.; Meng, Y.; Ding, S. Attribute-level and pattern-level classification consistency and accuracy indices for cognitive diagnostic assessment. J. Educ. Meas. 2015, 52, 457-476. [CrossRef]

82. Sinharay, S.; Johnson, M.S. Measures of agreement: Reliability, classification accuracy, and classification consistency. In Handbook of Diagnostic Classification Models; von Davier, M., Lee, Y.-S., Eds.; Springer International Publishing: Cham, Switzerland, 2019; pp. 359-377. [CrossRef]

83. Huebner, A.; Wang, C. A note on comparing examinee classification methods for cognitive diagnosis models. Educ. Psychol. Meas. 2011, 71, 407-419. [CrossRef]

84. Nakazawa, M. fmsb: Functions for Medical Statistics Book with Some Demographic Data; R Package Version 0.7.1. 2021. Available online: https: / /CRAN.R-project.org/package=fmsb (accessed on 14 September 2021).

85. Chiu, C.-Y.; Ma, W. ACTCD: Asymptotic Classification Theory for Cognitive Diagnosis; R Package Version 1.2-0. 2018. Available online: https:/ /CRAN.R-project.org/package=ACTCD (accessed on 9 September 2021). 